\title{
Évaluation de l'aléa glissements de terrain : état de l'art et perspectives pour la cartographie réglementaire en France
}

\author{
Yannick Thiery ${ }^{\star}$ et Monique Terrier \\ BRGM, Direction Risques et Prévention, Orléans, France
}

\begin{abstract}
Résumé - En France, dans le cadre de la cartographie réglementaire (type PPR - plans de prévention des risques), l'évaluation de l'aléa glissements de terrain suit généralement une approche empirique. Outre les données de base disponibles, les résultats sont donc étroitement dépendants de la qualité d'expertise réalisée. Depuis quelques années, dans un but opérationnel et afin de réduire les incertitudes de l'évaluation de l'aléa, des approches parallèles ou complémentaires ont été développées. Dans le cadre de cette article, un état de l'art sur l'approche cartographique actuelle est tracé, puis les perspectives et limites des approches en cours de développement sont décrites.
\end{abstract}

Mots clés : glissements de terrain / aléa / état de l'art / cartographie / approches alternatives

\begin{abstract}
Landslide hazard assessment: State of the art and perspectives for the regulatory zoning in France. In France, in the context of regulatory mapping (PPR - Risk Prevention plan based on French regulatory rules), landslide hazard assessment follows an empirical approach. In addition to the basic available data, the results are therefore closely linked to the quality of the expertise. Since few years, in order to reduce the hazard assessment uncertainties, alternative or complementary approaches have been developed. In the framework of this article, a state of the art on the current mapping approach is outlined, and then the perspectives and limitations of alternative approaches being developed are described.
\end{abstract}

Keywords: landslides / hazard / state of the art / mapping / alternative approaches

\section{Introduction}

Un glissement de terrain correspond au déplacement d'une masse de matériaux cohérents le long d'une surface de rupture sous l'effet de la gravité (Varnes, 1978). Phénomènes multiples (rotationnels, translationnels, mixtes) et complexes, associant un secteur de rupture en amont et un secteur d'accumulation en aval, ils peuvent être ponctuels, superficiels, lents, limités dans l'espace et dans le temps mais également rapides, et de grande ampleur affectant dans ce cas des versants entiers (Ledoux, 1995 ; Thiery, 2007). Actifs, latents, inactifs ou potentiels, ils se caractérisent par une répartition spatiale et temporelle assez diffuse et peuvent être localisés en régions montagneuses, mais également dans des régions à relief plus modéré (i.e. fronts des cuestas, versants des plateaux,...) présentant des facteurs de prédisposition favorables. Leur déclenchement peut être provoqué par des épisodes de précipitations intenses et/ou un fort cumul de précipitations, mais peut également se produire lors de périodes de fonte de neige, sous l'effet d'une vibration sismique, ou encore après une intervention humaine.

\footnotetext{
$\bar{\star}$ Auteur de correspondance : y.thiery@brgm.fr
}

Malgré les progrès réalisés depuis une trentaine d'années sur la compréhension des mécanismes à l'origine des glissements de terrain ainsi que sur les techniques de mitigation, en France, ces mouvements engendrent, en moyenne entre 2 et $3 \mathrm{M} € . \mathrm{an}^{-1}$ de dégâts directs (Grislain-Letrémy et Peinturier, 2010). Si les conséquences directes peuvent être évaluées relativement rapidement, les conséquences indirectes sont plus difficiles à estimer car dépassant le cadre local et étant parfois sur du long terme. Par exemple, la déstabilisation du versant du Chambon en 2015 a demandé $5 \mathrm{M} €$ pour l'aménagement de la piste en rive gauche, ainsi que $12 \mathrm{M} €$ pour des études géotechniques, la mise en place des navettes lacustres, etc. À ces coûts il faudra ajouter $24,4 \mathrm{M} €$ de travaux qui seront alloués pour la sécurisation de l'ancien tunnel et la construction d'un tunnel de dérivation. Toutefois, ces chiffres n'incluent pas les pertes économiques liées aux activités commerciales locales, l'impact financier pour les entreprises extérieures à la vallée ou encore les pertes relatives à l'activité touristique.

Un risque naturel majeur combine à la fois l'aléa, les enjeux et leur vulnérabilité. L'aléa est l'évènement naturel qui peut créer les dommages s'il se réalise. Il est caractérisé par un niveau d'intensité et une probabilité de survenance. Les enjeux concernent les personnes, les biens et activités susceptibles 


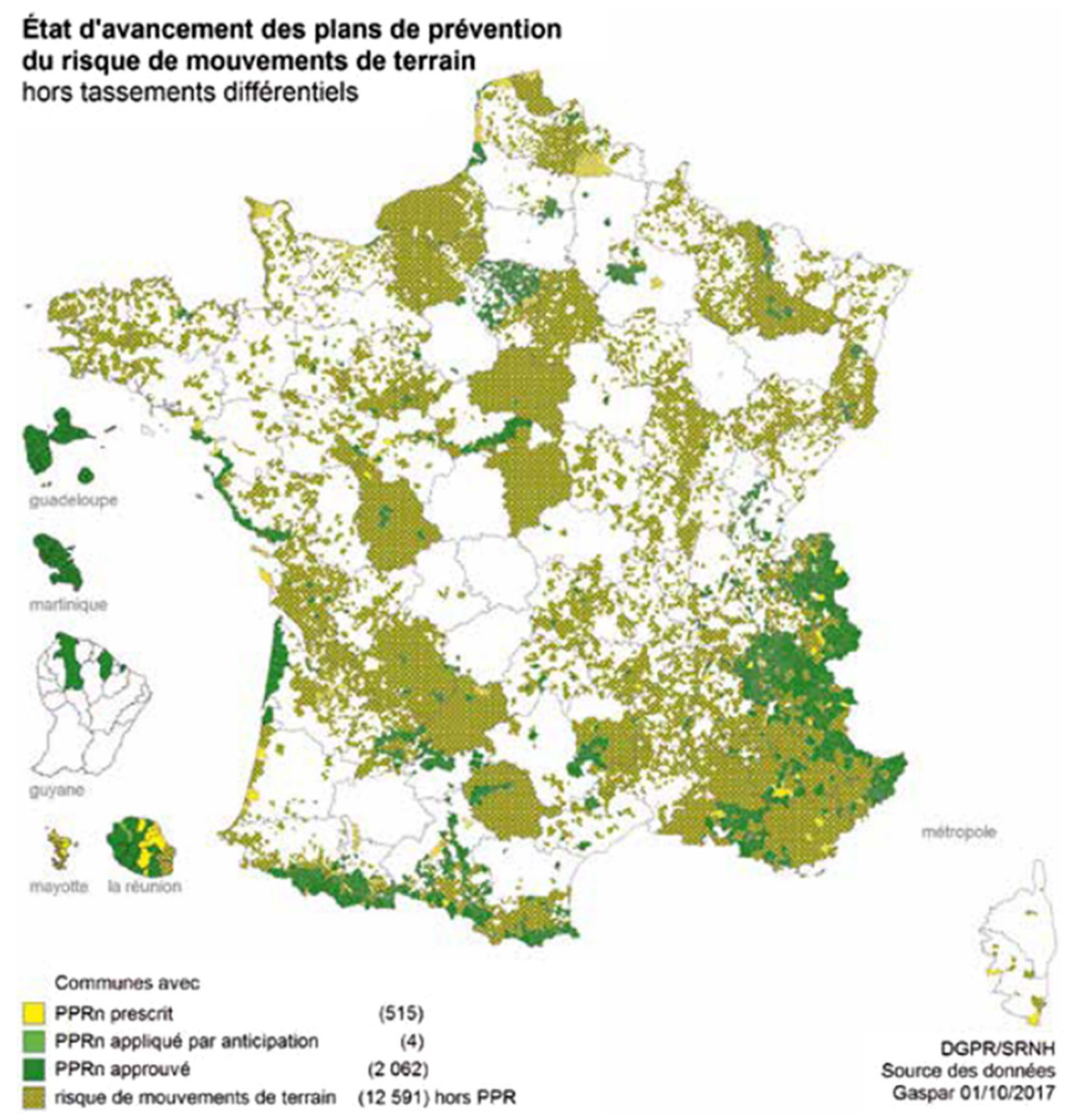

Fig. 1. État d'avancement des PPR mouvements de terrain en France au $1^{\text {er }}$ octobre 2017 (Source: base de données Gaspar).

Fig. 1. Progress status for landslide PPR (landslide risk maps based on regulatory procedure) for 2017 October 1st (origin: Gaspar database).

d'être affectés par l'évènement. Ils sont associés à une vulnérabilité qui indique un degré variable d'endommagement en cas d'évènement d'intensité donnée.

Dans une volonté de réduire les risques naturels sur son territoire, la France a engagé depuis plusieurs décennies une politique de prévention des risques naturels. Dans le panel des moyens développés, la cartographie et la réglementation des zones à risques constitue un outil fondamental pour la prise en compte des risques dans l'aménagement, la construction et la gestion d'un territoire communal (Loi $\mathrm{n}^{\circ} 82-600 \mathrm{du} 13$ juillet 1982 et Loi n ${ }^{\circ} 95-101$ du 2 février 1995). Établis dans le cadre législatif, les plans de prévention des risques naturels (PPRN) définissent les zones d'exposition aux phénomènes naturels prévisibles, directs et indirects, et caractérisent l'intensité possible de ces phénomènes pour différents aléas. Le règlement du PPRN accompagne la carte règlementaire. En fonction de l'aléa et de l'occupation du sol (actuelle ou à venir), il précise les règles devant être suivies afin de ne pas augmenter les enjeux dans les zones à risques et de diminuer la vulnérabilité des zones déjà urbanisées. Le PPRN doit permettre d'orienter le développement d'une commune vers les zones les moins exposées aux aléas naturels. En octobre
2017, sur les 12591 communes exposées, 2062 communes étaient couvertes par un PPRN approuvé (Fig. 1). Dans le cadre des PPR, cette évaluation s'effectue très généralement selon une démarche empirique, de type «expert», fondée sur les données disponibles au regard des connaissances du moment et sur les connaissances de l'expert (Antoine et al., 2000). Ainsi, ce dernier définit le(s) type(s) de phénomène(s) puis les rôles respectifs des facteurs de prédisposition et de déclenchement pour placer une limite estimée la plus pertinente pour les 100 prochaines années. Cependant, la démarche reste difficile et est sujette à de nombreuses limites liées notamment : (i) à la nature discontinue dans le temps et l'espace des phénomènes ; (ii) à la qualité des informations disponibles (données historiques fiables et complètes, données spatiales incomplètes, etc.); (iii) aux difficultés d'identifier les causes des phénomènes; (iv) aux erreurs d'appréciation inhérente à la personne en charge du dossier et son degré d'expertise.

Depuis une quinzaine d'années la démocratisation des approches cartographiques sous SIG (système d'information géographique); le développement de nouvelles techniques pour acquérir des données topographiques, du sol et du soussol; la baisse des coûts d'acquisition des données spatiales de 
haute et de très haute résolution; et la mise à disposition de certaines bases de données spatiales dédiées aux géosciences offrent des possibilités pour réduire les incertitudes liées à ces limites. Ainsi, actuellement une réflexion doit être menée sur les manières d'améliorer l'évaluation de l'aléa glissement de terrain et sur les outils disponibles qui peuvent contribuer à améliorer les cartes d'aléa. Après avoir établi un état de l'art sur les approches spatiales numériques et dressé une liste non exhaustive d'outils et techniques émergents, cet article se focalisera sur les perspectives qu'ils offrent pour la cartographie de l'aléa glissement de terrain dans un cadre réglementaire comme les PPR.

\section{2 Évaluation de l'aléa glissement de terrain: état de l'art}

L'évaluation de l'aléa revient à répondre à ces quatre questions : où, quand, comment et pourquoi le phénomène se produira? Ainsi, Cela revient à évaluer la probabilité d'occurrence spatiale (susceptibilité: probabilité spatiale qu'un type de phénomène se produise sur un territoire pour différentes conditions environnementales locales), temporelle (période de retour) et de propagation d'un phénomène d'intensité donnée (Cruden et Fell, 1997; Antoine et al., 2000 ; Fell et al., 2005). Cela suppose que tous les phénomènes soient identifiés et classés (inventaire) et qu'ils se (re) produiront selon les mêmes conditions géologiques, géomorphologiques, hydrologiques et climatiques que les phénomènes connus.

Cependant, quantifier un niveau d'aléa reste un exercice difficile. En effet, les données nécessaires à une analyse complète et rigoureuse (incluant facteurs de déclenchement, date de déclenchement, âge, etc...) ne sont pas toujours disponibles et/ou insuffisamment détaillées. Par conséquent, fréquemment à l'échelle du bassin versant, seule la susceptibilité des terrains à un type de phénomène est analysée, on parle alors d'une évaluation relative de l'aléa (Wu et al., 1996; Corominas et al., 2014).

\subsection{Le zonage réglementaire en France}

\subsubsection{Historique}

Les cartes ZERMOS (zones exposées aux risques de mouvements du sol et du sous-sol ; Fig. 2A) furent les premiers essais de cartographie informative ou d'orientation pour les zones exposées à des risques de mouvements du sol et du soussol (Humbert, 1977). Engagées dès 1971, ces cartes visaient à constituer des documents de synthèse sur la probabilité spatiotemporelle d'occurrence des phénomènes à l'échelle du $1: 25000^{\text {ème }}$ (Humbert, 1977). La méthodologie mise en œuvre est qualitative avec des observations de terrain systématiques et un zonage réalisé de manière experte (Antoine et al., 2010). Ces cartes pouvaient servir de base au POS-ZERMOS définissant des niveaux de contraintes face aux phénomènes naturels à l'échelle du plan d'cccupation des sols (1:5000 ${ }^{\text {ème }}$; Guillope et Porcher, 1979).

En 1982, sont mis en œuvre les plans d'exposition aux risques naturels prévisibles (Loi $\mathrm{n}^{\mathrm{o}} 82-600$; Fig. 2B) ce qui relance la question de la cartographie des risques. Le PER était un document technique synthétique visant à être appliqué pour différents types de phénomènes naturels (crues, mouvements de terrain, séismes et avalanches). Il n'a plus seulement une valeur informative ou consultative mais constitue un document opposable aux tiers, imposant des contraintes pour le développement territorial des communes concernées (Fressard, 2013). Il ajoute également la notion de prévisibilité n'interdisant pas l'occurrence d'un phénomène dans un contexte où rien ne laissait présager cette dernière. Quatre documents principaux sont produits : une carte informative des phénomènes, une carte d'aléa, une carte de vulnérabilité et un plan d'exposition aux risques qui est restitué à l'échelle du $1: 5000^{\text {ème }}$. Le principe méthodologique est le même que pour les cartes ZERMOS (Champetier de Ribes, 1987), c'est-à-dire, fondé sur une analyse qualitative dite experte.

\subsubsection{Les PPR}

Les PER sont remplacés par les plans de prévention des risques naturels prévisibles (PPR; Fig. 2C) avec la loi du 2 février 1995, (Loi n ${ }^{\circ} 95-101$ ) avec une volonté de simplifier et homogénéiser les procédures de cartographie réglementaire (PER) qui se révélaient être complexes, lourdes et rigides (MATE/MATL, 1997; Maquaire, 2002). Comme pour les PER, quatre documents graphiques sont produits avec: une carte informative des phénomènes, une carte d'aléa, une carte des enjeux et un plan de zonage PPR ; ainsi qu'un un règlement comprenant les mesures à prendre selon les zones retenues. D'un point de vue de l'évaluation et de la cartographie de l'aléa glissement de terrain, les PPR reprennent les mêmes recommandations que les PER avec une évaluation qualitative privilégiant l'utilisation et les connaissances du moment. Quelques modifications sont tout de même à noter d'un point de vue des ensembles à cartographier: (i) la notion de bassin de risque sera appliquée plutôt que l'ensemble d'un territoire communal; (ii) l'échelle de travail privilégiée sera le 1: $10000^{\text {ème }}$ avec une certaine flexibilité permettant de limiter les investigations en cas d'aléa faible et/ou modéré; (iii) le caractère temporel n'est plus figé dans le temps ou l'espace permettant des modifications (révision) si de nouveaux éléments viennent à être portés à connaissance. Par ailleurs, la qualification de l'intensité de l'aléa est non seulement fonction de la typologie du mouvement de terrain, des conditions géologiques et topographiques, mais aussi il est recommandé de tenir compte de l'ampleur estimée du (ou des) phénomène(s) d'instabilités, et du niveau d'importance des parades à mettre en place pour stabiliser les terrains.

\subsection{Méthodes d'évaluation spatiale de l'aléa glissement de terrain}

Si la méthodologie de cartographie de l'aléa pour les PPR est qualitative, il existe d'autres méthodes d'évaluation de l'aléa glissement de terrain mobilisant différentes disciplines comme la géomorphologie, la géologie, l'hydrogéologie, les mathématiques, la statistique, ou encore la géotechnique (Thiery, 2007; Fressard, 2013 ; Corominas et al., 2014). Si certaines de ces méthodes ont bénéficié d'une augmentation croissante des capacités de calcul informatique, toutes ont le même objectif et sont fondées sur la relation entre les glissements de terrain existants et les facteurs environnementaux du site étudié. Ainsi, chacune des méthodes doit bénéficier 

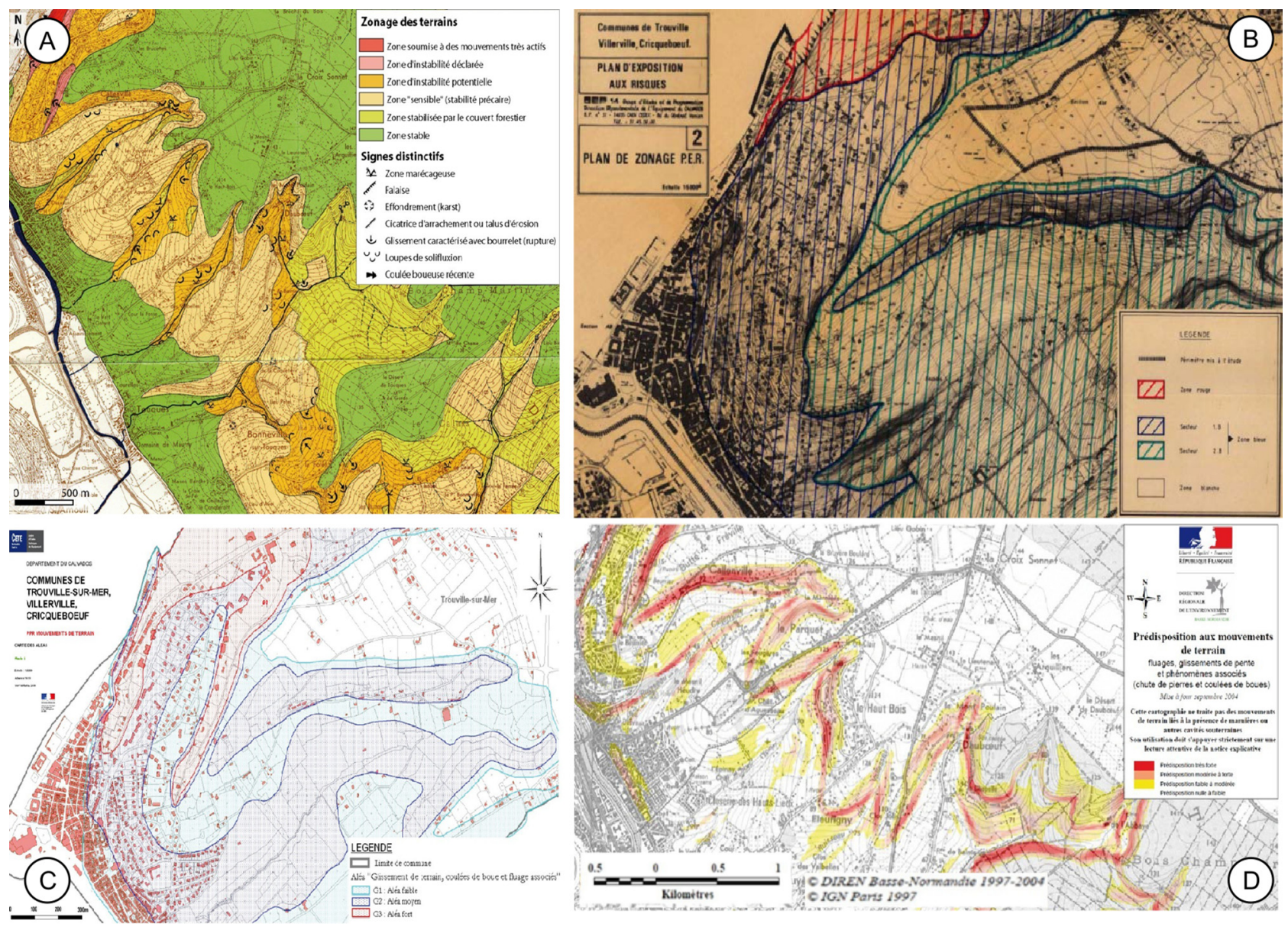

Fig. 2. Extraits de cartes d'aléa réglementaires produites pour différents zonages réglementaires. A. Extrait de carte d'aléa ZERMOS pour Trouville/Pont l'Evêque (BRGM, 1976). B. Extrait du plan d'exposition aux risques (PER) Trouville/Villerville-Criqueboeuf (LCPC, Rouen, 1986). C. Extrait de la carte d'aléa au $1: 10000^{\text {ème }}$ du PPR de Trouville/Villerville-Cricquebeuf (DDTM, validation le 24 avril 2009). D. Extrait de la carte de susceptibilité à différents glissements de terrain, carte produite par combinaison lithologie/pente (DREAL, 2004).

Fig. 2. Extracts of regulatory hazard maps produced for different regulatory zoning. A. Extract of ZERMOS hazard map for Trouville/Pont l'Evêque (BRGM, 1976). B. Extract of exposed risk map (PER) for Trouville/Villerville-Criqueboeuf (LCPC, Rouen, 1986). C. Extract from the hazard map at 1:10 000 of the Trouville/Villerville-Cricquebeuf based on PPR procedure (DDTM, validated on 24 April 2009). D. Extract from the landslide susceptibility map produced by combination of lithology and slope (DREAL, 2004).

d'une source d'information commune nécessaire et indispensable: l'inventaire des phénomènes (Fig. 3). Une synthèse exhaustive de ces méthodes peut être trouvée dans Soeters et van Westen (1996), Thiery (2007), Fressard (2013), et Corominas et al. (2014).

\subsubsection{Une base commune: l'inventaire des phénomènes}

L'inventaire des phénomènes sur un territoire (bassinversant, commune, région, etc.) peut être obtenu (i) soit de manière conventionnelle par observations de terrain, analyse de documents existants, télédétection (analyses visuelles); (ii) soit par techniques semi-automatique ou automatique de télédétection (Cascini, 2008; Guzzetti et al., 2012). Le minimum d'information requis est le type de phénomène, son degré d'activité, son âge (si possible), ses paramètres morphométriques et les conditions de déclenchement. À partir de cartes d'inventaire il est possible de construire des cartes indicatives comme des cartes d'activité ou de densité de glissements de terrain (Fig. 3, Soeters et van Westen, 1996).
Néanmoins, sans inventaire, il reste hasardeux de construire une carte d'aléa et de fortes incertitudes inhérentes aux manque d'information subsisteront sur les documents finaux (Maquaire, 2002). Ainsi, l'inventaire des phénomènes est un prérequis pour toute évaluation de la susceptibilité et de l'aléa quelles que soient l'échelle d'analyse et l'approche retenue (Cascini, 2008; Fell et al., 2008; Corominas et al., 2014).

\subsubsection{Méthodes qualitatives ou empiriques}

Les méthodes qualitatives (ou empiriques) se divisent en deux catégories avec: (i) la méthode "géomorphologique» dite directe et (ii) les méthodes indirectes (Fig. 3).

La méthode directe : est fondée sur une analyse géologique et géomorphologique des terrains. Ce type de méthode a été utilisée pour la cartographie de type ZERMOS et peut être utilisée pour la cartographe réglementaire. L'évaluation et/ou le zonage est réalisé sur le terrain par l'expert qui, sur la base de ses observations et de son expérience, placera les limites des secteurs cartographiés et un degré d'aléa estimé. Ainsi, 


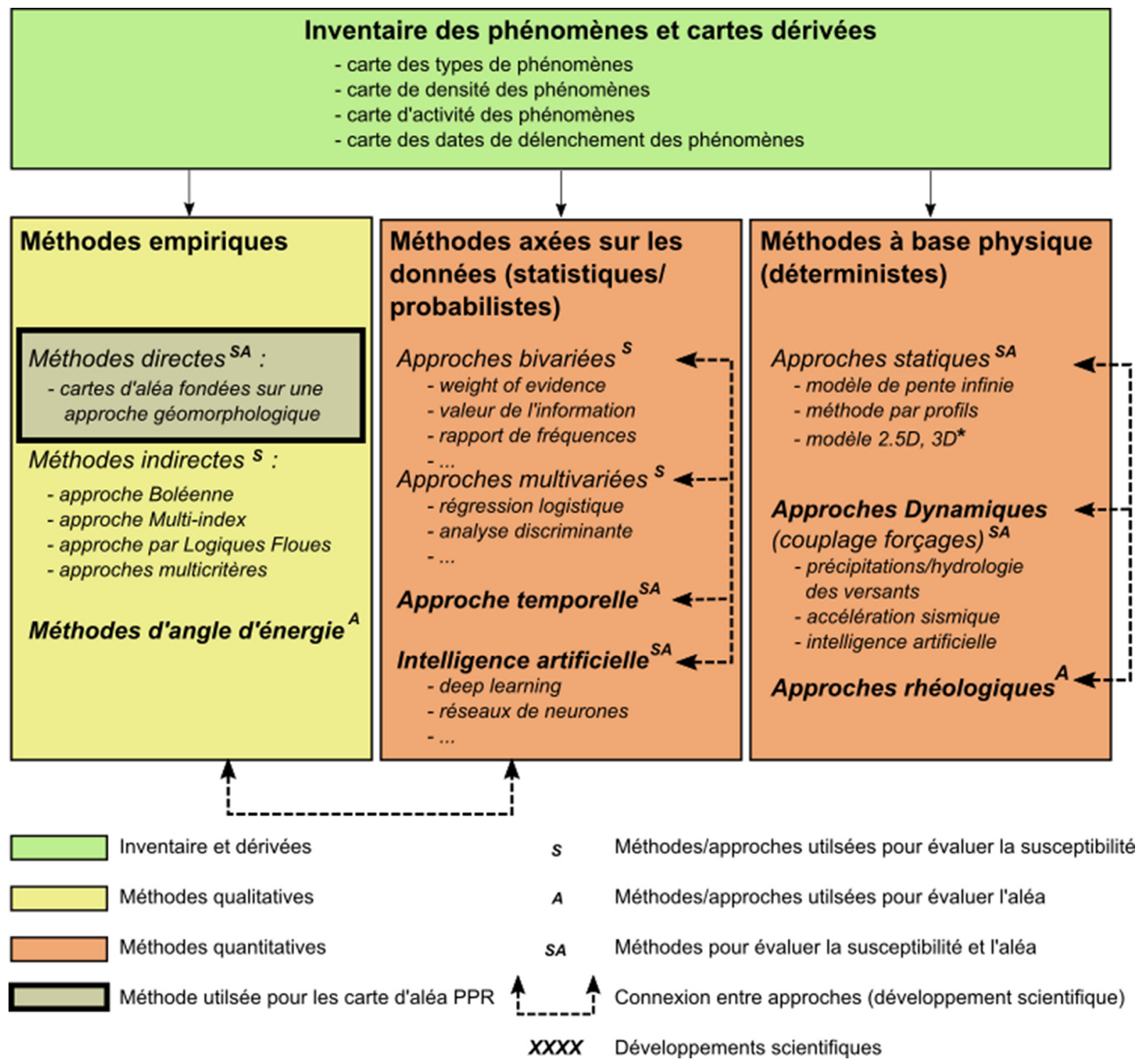

Fig. 3. Méthodes pour évaluer la susceptibilité et l'aléa glissement de terrain (d'après Soeters et van Westen, 1996; van Westen et al., 2006 ; Fell et al., 2008; Corominas et al., 2014).

Fig. 3. Methods for landslide susceptibility and hazard assessement (from Soeters et van Westen, 1996; van Westen et al., 2006; Fell et al., 2008; Corominas et al., 2014).

l'expert synthétise directement les informations et peut intégrer un grand nombre de facteurs. Les avantages de la méthode sont sa rapidité de mise en œuvre et l'intégration de la propagation des terrains glissés dans les enveloppes cartographiées. Les défauts majeurs résident dans une démarche peu expliquée avec des règles implicites difficilement reproductibles par autrui (van Westen et al., 2008). Les systèmes d'information géographique (SIG) sont utilisés comme outil de vectorisation pour la carte d'aléa finale (Thiery, 2007).

Les méthodes indirectes: contrairement à la méthode directe, l'expert tente de rationaliser et d'expliciter son raisonnement et de quantifier chaque facteur contributif, ce type de méthode peut être utilisée pour la cartographie réglementaire. Plusieurs méthodes existent; toutes partent du même principe qui consiste à : (i) sélectionner les phénomènes ; (ii) sélectionner les facteurs contributifs (sous forme de variables spatiales); (iii) attribuer un poids relatif pour chaque facteur de prédisposition et classe respective (chaque poids étant proportionnel à la contribution attendue par l'expert pour générer un type de phénomène); (iv) combiner sous SIG les variables afin d'obtenir, après reclassification, des zones homogènes favorables ou pas aux glissements de terrain. Plusieurs approches sont distinguées avec: (i) l'approche par logique booléenne; (ii) l'approche par combinaison de cartes d'index; (iii) l'approche par logiques floues; (iv) les systèmes multicritères (analyse par processus hiérarchique; Saaty, 1977). Les deux dernières citées sont en termes de combinaison de variables et de formalisation de règles expertes les plus complètes. De plus, elles gardent la souplesse de l'approche géomorphologique tout en étant considérées comme plus objectives par le cadre formaliste qu'elles imposent à leur application (Poiraud, 2012). Pour ces méthodes, seule la susceptibilité est évaluée. 


\subsubsection{Méthodes quantitatives}

Par opposition aux méthodes qualitatives, les méthodes quantitatives sont considérées comme objectives. Elles sont théoriquement reproductibles pour des environnements similaires en produisant des résultats identiques avec un même jeu de variables (Thiery, 2007; Fressard, 2013). Deux grands types de méthodes quantitatives sont différenciées (Fig. 2): (i) les méthodes statistiques/probabilistes (ou méthodes axées sur les données) et (ii) les méthodes déterministes (ou à base physique).

Les méthodes statistiques/probabilistes sont celles qui ont connues le plus grand essor depuis la deuxième moitié des années 1990 (Reichenbach et al., 2018). Il est possible d'utiliser plusieurs approches: les approches bivariées le plus souvent fondées sur le théorème de Bayes (Weight of Evidence, valeur de l'information, rapport de fréquence, etc.); les approches multivariées (régression logistique, analyses discriminantes, ...): les approches par réseaux de neurones artificiels. Le principe est le même pour chacune, il repose sur la distribution spatiale des phénomènes et une comparaison avec les différents facteurs (sous forme de variable spatiale). Ainsi, selon l'approche choisie, une pondération pour chaque classe de facteur est obtenue. Les pondérations sont donc définies de manière objective, sans intervention de l'expert, puis comme pour les méthodes qualitatives indirectes une combinaison sous SIG est réalisée. Le zonage en secteurs homogènes favorables ou pas aux glissements de terrain est effectué après reclassification des modèles. Si les résultats sont robustes et peuvent être transposés à d'autres sites semblables (à condition de bien caler les approches), ces méthodes axées sur les données, comme pour les méthodes indirectes, se focalisent sur l'analyse de la susceptibilité et peu intègrent une notion temporelle. Lorsque cette dernière est intégrée c'est soit sous forme de calcul de probabilité spatiale de retour des phénomènes (Crovelli, 2000; Coe et al., 2004) ou soit en couplant des analyses statistiques des facteurs de déclenchement (précipitation) et susceptibilité (Zêzere et al., 2004) ce qui demande des inventaires exhaustifs avec les dates de déclenchement des phénomènes.

Les méthodes à base physique sont fondées sur des modèles de calcul d'équilibre limite (calcul du facteur de sécurité-FS). Deux types d'approche sont généralement utilisées (Fig. 2): les approches statiques et les approches dynamiques. Les calculs sont réalisés en 2D, 2,5D parfois en 3D pour un rendu cartographique 2D (Mergili et al., 2014; Dang et al., 2016). Elles reposent pour la majorité sur des modèles de pentes infinies, plus rarement sur des modèles de rupture complexe (Thiery et al., 2017a, 2019). Pour les deux approches, les facteurs de déclenchement (précipitations, niveau de saturation des matériaux, accélération sismique) sont pris en compte. Pour l'approche dynamique, il est possible de faire de la prévision en tenant compte de chroniques et de changements temporel du paysage et/ou des facteurs de déclenchement. Ainsi, par rapport aux approches décrites précédemment, ces méthodes demandent des informations supplémentaires comme des données hydrologiques (saturation des sols, perméabilité, conductivité hydraulique, etc.) et géotechniques (épaisseur des matériaux, cohésion, angle de frottement interne, poids spécifique etc.). Contrairement aux méthodes statistiques/probabilistes, ces méthodes sont considérées comme plus concrètes et moins exploratoires, les processus physiques étant intégrés et des valeurs quantitatives de stabilité calculées (Corominas et al., 2014). Toutefois, au regard du grand nombre d'informations requises, elles ne sont appliquées que sur des petits bassinversants ou sur un phénomène particulier, ce qui les rend difficilement transposables. En effet, une certaine généralisation des données d'entrée demanderait des approximations engendrant des imprécisions des résultats (Zizioli et al., 2013; Thiery et al., 2019). Enfin, leur paramétrisation peut s'avérer complexe pour les non-initiés.

\subsubsection{Les récentes avancées en terme d'évaluation spatiale de l'aléa glissement de terrain}

Depuis quelques années de nouveaux développements émergent pour améliorer l'évaluation de la susceptibilité et de l'aléa afin de réduire les incertitudes, augmenter le pouvoir prédictif des modèles, ajuster le zonage et tenir compte de la propagation des phénomènes. Ces développements spécifiques sont fondés sur des algorithmes utilisés dans d'autres disciplines (van den Eeckhaut et al., 2006), des stratégies spécifiques adaptées aux sites (van Westen et al., 2003; Thiery et al., 2007, 2014, Fig. 2), sur le développement de modèles complexes validés mathématiquement, calés sur un évènement, mais qui restent à tester sur un bassin de risque (Corominas et al., 2014).

Pour l'évaluation de la susceptibilité, outre des modifications mathématiques d'approches éprouvées (comme l'utilisation des régressions logistiques pour évènements rares; van den Eeckhaut et al., 2006; Fressard et al., 2014) permettant d'améliorer le pouvoir prédictif des modèles, des tests de méthodes issues du data-mining et de l'intelligence artificielle s'intensifient avec des résultats prometteurs (Chen et al., 2016, 2017; Pham et al., 2017).

Pour l'évaluation de l'aléa, au-delà des modèles à base physique d'équilibre limite qui se multiplient et qui tiennent compte des incertitudes environnementales ainsi que différents types de rupture (Mergili et al., 2014; Thiery et al., 2017a), certains développements s'effectuent sur la propagation. Ainsi deux axes sont privilégiés : (i) un axe fondé sur des approches empiriques tenant compte de la distance de propagation maximum des phénomènes et de l'énergie selon les angles de pentes(Horton et al., 2013); (ii) un axe fondé sur des approches numériques modélisant le comportement des matériaux en mouvement selon certaines lois rhéologiques (Pastor et al., 2014).

Le premier axe est plutôt utilisé comme une aide à la cartographie (Sedan, 2011; Horton et al., 2013); les épaisseurs, les vitesses et les caractéristiques géotechniques des matériaux n'étant pas intégrées. Le second axe est plus exploratoire et nécessite encore des développements notamment pour les glissements à contrôle hydrologique et au comportement complexe (visco-élastique; visco-élasto-plastique) et sur la manière de les utiliser sur des bassins de risques.

\subsection{Pour chaque approche un jeu de données et une échelle adaptés}

Cet état de l'art ne serait pas complet si la question des données d'entrées (variables), leur résolution et leur qualité n'étaient pas discutées. Les glissements de terrain sont 
Tableau 1. Classification des différents niveaux de zonage de la susceptibilité aux glissements de terrain en fonction des méthodes et données utilisées (d'après Cascini, 2008; Fressard, 2013).

Table 1. Classification for different landslide susceptibility assessment levels according to the methods and data used (from Cascini, 2008; Fressard, 2013).

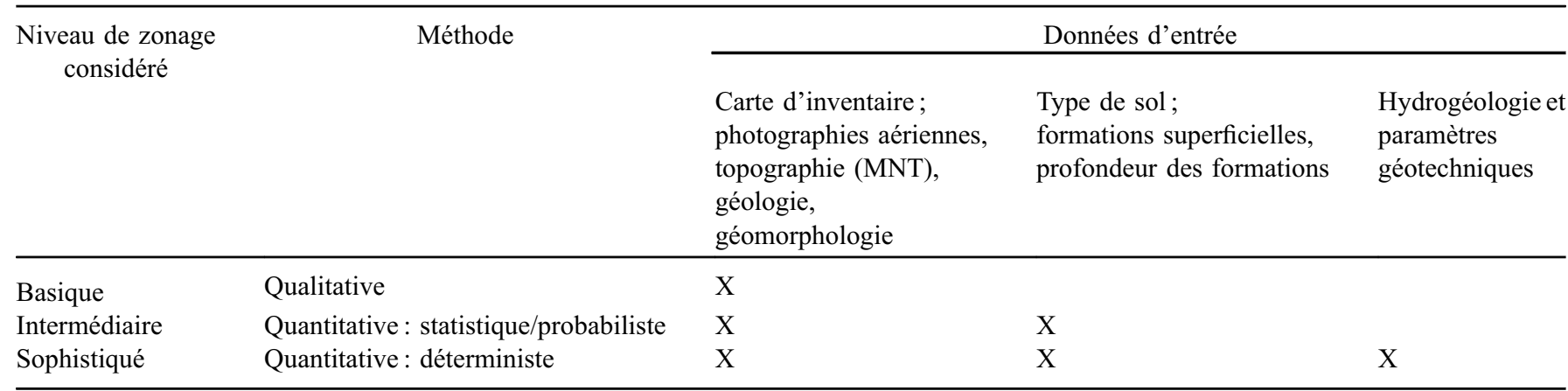

Tableau 2. Classification des différents niveaux de zonage de l'aléa glissement de terrain en fonction des méthodes et données utilisées (d'après Cascini, 2008; Fressard, 2013).

Table 2. Classification for different landslide hazard assessment levels according to the methods and data used (from Cascini, 2008; Fressard, 2013).

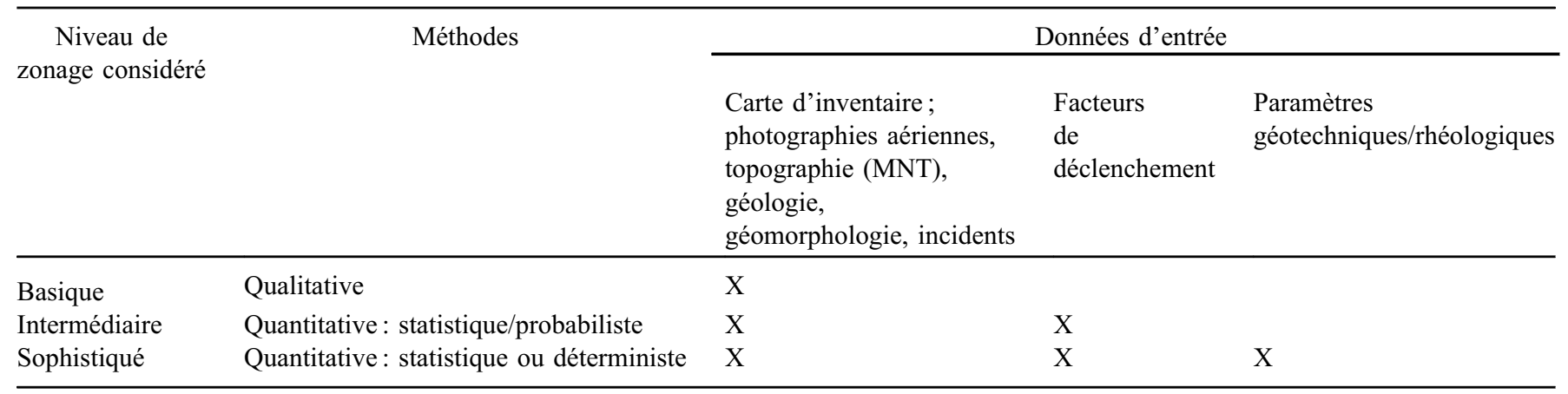

commandés par une/des combinaison(s) de plusieurs facteurs qui doivent être analysés simultanément afin d'obtenir des résultats corrects et valides (Thiery, 2007 ; Fressard, 2013). Du nombre de données et de leur qualité dépend le type de méthode et d'approche envisagée et donc du problème considéré (en d'autres mots : traitera-t-on de la susceptibilité ou de l'aléa ?; Aleotti et Chowdhury, 1999; Glade et Crozier, 2005). Plusieurs classifications tenant compte de ces paramètres ont été proposées (Cascini, 2008; Fell et al., 2008; Corominas et al., 2014) aboutissant à un niveau de zonage basique, intermédiaire ou complexe (Tab. 1 et 2). Ainsi, en fonction $\mathrm{du}$ jeu de données initial, une méthode et une approche seront choisies. Comme il n'est pas toujours possible d'avoir un jeu de données optimum, de grande qualité et de haute résolution (problèmes de coûts et de temps, données non disponibles et/ou demandant un temps long d'acquisition, etc.), il est préférable de disposer de quelques données de qualité et d'envisager un zonage basique qui pourra être justifié et probant plutôt qu'une multitude d'information de qualité médiocre et un zonage complexe avec de fortes incertitudes.

Enfin, il faut également considérer l'échelle de travail qui est fonction des données acquises et disponibles. Ainsi, il est généralement admis qu'aux petites échelles seules les méthodes qualitatives directes peuvent-être utilisées et qu'aux grandes échelles les méthodes déterministes sont recommandées. Ce constat est à nuancer en fonction de la méthode choisie et de la qualité des données. Par exemple, il est possible, lorsque l'environnement s'y prête (environnement homogène), de traiter l'aléa aux échelles du $1: 25000^{\text {ème }}$ sur de grands secteurs (Salciarini et al., 2006) ou encore d'utiliser des approches quantitatives statistiques à une échelle du $1: 10000^{\text {ème }}$ (Thiery et al., 2007). Le tableau 3 donne un aperçu synthétique des méthodes, des niveaux de zonage et des échelles qui sont généralement recommandées.

\section{Les techniques et données émergentes pour une amélioration de la connaissance des phénomènes et de leur cartographie}

Comme il est précisé précédemment la qualité des données conditionne l'approche d'évaluation de l'aléa. Depuis quelques années on assiste à une multiplication des techniques qui permettent d'acquérir rapidement des données nécessaires pour cartographier les phénomènes, obtenir de l'information précise sur la topographie ou encore sur la structure interne du sol et du sous-sol complétant ainsi les données disponibles ou déjà acquises. Trois grandes thématiques ressortent avec les progrès réalisés (i) en télédétection (photographies aériennes et images satellites); (ii) en géophysique;(iii) les outils de cartographie de terrain. 
Tableau 3. Classification des méthodes, de leur précision et du champ d'application selon l'échelle de travail (d'après Cascini, 2008). Table 3. Classification of methods, their accuracy and their scope according to work scale (from Cascini, 2008).

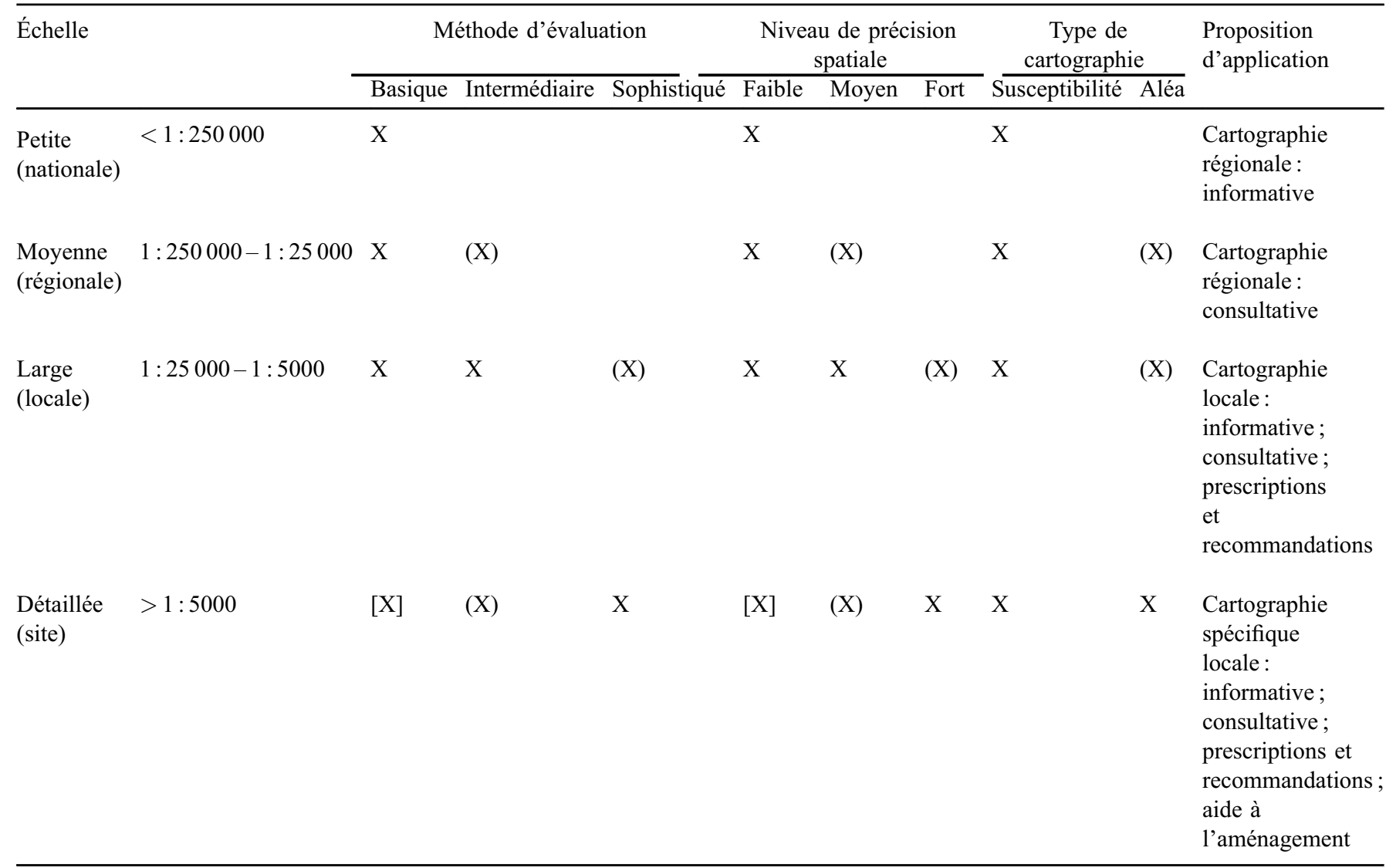

Note: $\mathrm{X}=$ recommandée; $(\mathrm{X})=$ applicable avec précautions ou développements spécifiques; $[\mathrm{X}]=$ non recommandé.

$X=$ recommended; $(X)=$ applicable with specific precautions or developments; $[X]=$ not recommended.

\subsection{Techniques et données issues de la télédétection}

Les techniques et données issues de la télédétection ont été divisées en deux groupes selon l'apport pour notre problématique avec: (i) l'utilisation des MNT haute résolution obtenus par différentes techniques et (ii) l'utilisation d'images satellites (panchromatique, multi-spectrale ou radar) pour identifier les formes et/ou l'activité des phénomènes.

\subsubsection{Les MNT haute résolution pour analyser les formes}

Les récents développements, la mise à disposition et la baisse des coûts des MNT très haute résolution obtenus par laser aéroporté ou terrestre, ou encore par drones équipés de capteurs optiques très haute résolution, ont donné aux personnes en charge de cartographier les glissements de terrain des possibilités de détection des formes et des phénomènes instables encore jusque-là inégalées (van den Eeckhaut et al., 2005 ; Guzzetti et al., 2012). Que ce soit après un traitement de points (pour les techniques laser) ou par photogrammétrie, les MNT obtenus permettent des analyses visuelles précises ou des traitements semi-automatiques ou automatiques de reconnaissance de formes (calcul d'indice de rugosité, identification de texture spécifique etc.; Sato et al., 2007; Iwahashi et Pike, 2007) facilitant (ou remettant en cause) certaines interprétations géomorphologiques réalisées sur le terrain. Par exemple, la figure 4 présente la différence entre trois MNT obtenus de manière différente et l'apport du LIDAR aéroporté en terme de résolution pour la reconnaissance des formes. La mise à disposition de MNT haute résolution comme le WorldDEM, la BDAlti $5 \mathrm{~m}$, ou BDAlti 1 $\mathrm{m}$ devrait encore accroître leur utilisation. Enfin, des acquisitions multi-temporelles de MNT haute résolution permettent d'observer des changements subtils de morphologie affinant la reconnaissance du degré d'activité de glissements de terrain actifs déterminé de manière experte.

\subsubsection{Les images satellites pour préciser les déformations du sol}

Outre des changements morphologiques, les glissements de terrain actifs, ou fraîchement déclenchés, modifient l'occupation du sol et par conséquent la signature spectrale du sol. Ainsi, il est possible avec les capteurs embarqués à bord de satellites de les détecter. Cette détection n'est pas nouvelle en soi (les premières analyses remontent aux années 1970 avec 

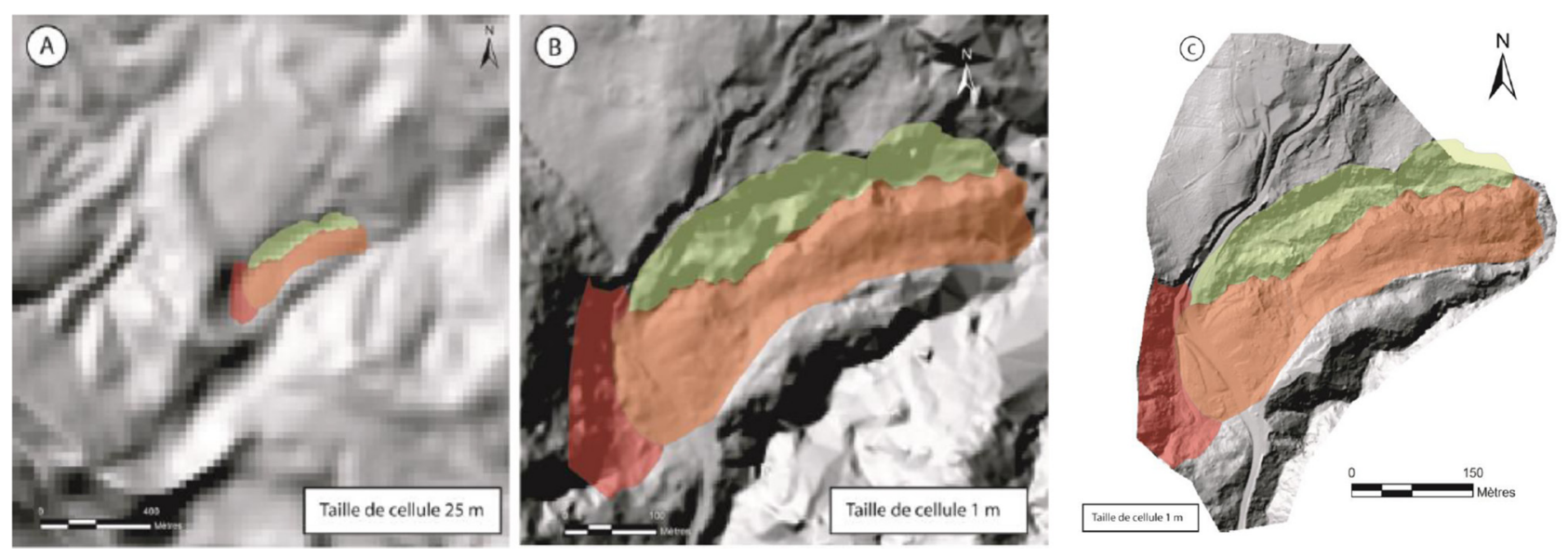

Fig. 4. Exemple de vues en relief dérivées de MNT obtenus par trois technologies différentes sur le site de la Médaille en Martinique (Thiery et al., 2015). A. BDAlti ${ }^{\circledR} 25 \mathrm{~m}$. B. Litto3D ${ }^{\circledR}$ (obtenu par LiDAR, résolution altimétrique $20 \mathrm{~cm} /$ résolution planimétrique $1 \mathrm{~m}$ ). C. MNT obtenu par technologie LiDAR créé spécifiquement pour le site (résolution altimétrique $10 \mathrm{~cm} /$ résolution planimétrique $0,20 \mathrm{~m}$-ré-échantillonné pour l'étude).

Fig. 4. Example of hillshaded views derived from DTMs obtained by three different technologies in La Médaille (Martinique; Thiery et al., 2015). A. BDAlti $25 \mathrm{~m}$. B. Litto3D (obtained by LiDAR, elevation resolution $20 \mathrm{~cm} /$ planimetric resolution $1 \mathrm{~m}$ ). C. DTM obtained by LiDAR technology created specifically for the site (elevation resolution $10 \mathrm{~cm} /$ planimetric resolution $0.20 \mathrm{~m}$-re-sampled for the study).

les images Landsat et SPOT) mais la résolution plus fine ainsi que les capacités numériques (augmentation du stockage de données, des capacités de calculs, de traitements, de visualisation des images) permettent de détecter des évènements qui auparavant ne l'étaient pas pour des secteurs peu accessibles. Guzzetti et al. (2012), après avoir procédé à une revue de littérature scientifique exhaustive, concluent que, pour la détection des glissements de terrain, les images haute résolution et très haute résolution (e.g. Spot, Ikonos, GeoEye, Sentinel...) issues des capteurs optiques (panchromatiques ou multi-spectraux) sont très largement utilisées alors que pour détecter les déformations lentes, les images radar (Synthetic Aperture Radar-SAR) sont privilégiées (e.g. ENVISAT, TerraSAR-X, TanDEM-X, Sentinel, ALOS/PALSAR; Fig. 5).

\subsection{Techniques et données issues de la géophysique}

Les techniques et données issues de la géophysique pour ausculter les versants instables sont massivement utilisées pour reconnaître la structure interne de glissements de terrain, les formations superficielles ou encore détecter des phénomènes instables (Frappa et Lebourg, 2001 ; Jongmans et Garambois, 2007). Trois techniques récentes se détachent actuellement pour leur potentiel d'application: (i) l'électromagnétisme héliportée (dispositif héliporté TDEM); (ii) L'imagerie 3D par tomographie sismique et inter-corrélation; (iii) la détection sismique automatique de glissements de terrain.

\subsubsection{L'électromagnétisme héliporté TDEM}

La méthode d'acquisition des données sur les formations du sol et du sous-sol par HTEM (dispositif héliporté TDEM) (Fig. 6) est relativement récente (Reninger, 2012). Combinant les avantages du HFEM (système héliporté en domaine fréquentiel; Dighem ${ }^{\circledR}$; ayant une bonne résolution latérale en proche surface quelle que soit la topographie) et du FTEM (système temporel «fixed-wing»; GEOTEM ${ }^{\circledR}$, QUESTEM ${ }^{(B)}$,
SPECTREM $^{\circledR}$; possédant une bonne profondeur d'investigation), ce système permet de couvrir rapidement de grandes surfaces, des sites peu accessibles aux topographies très escarpées avec une très bonne précision pour les épaisseurs des formations jusqu'à $250 \mathrm{~m}$ de profondeur (Reninger, 2012). Un autre avantage est d'éviter de multiplier les tests au sol réduisant ainsi certains coûts. Appliquée récemment en domaine volcanique particulièrement complexe (succession de laves aux caractéristiques hétérogènes) pour caractériser les formations superficielles (régolithe), la méthode a permis également de caractériser en 3D des formations de laves altérées favorables aux glissements de terrain, qu'ils soient superficiels ou profonds et d'apporter une aide dans le cadre de projets d'évaluation de l'aléa glissement de terrain (Reninger et al., 2014; Thiery et al., 2015, 2016, 2017b).

\subsubsection{Imagerie 3D par tomographie sismique et inter- corrélation}

La tomographie sismique permet de caractériser la structure interne d'un glissement de terrain ou de formations superficielles en localisant des contrastes de vitesses de propagation d'ondes sismiques (Samyn et al., 2011). La résolution dépend de la distribution des sources souvent difficiles à mettre en œuvre pour les sources actives. Toutefois, il est possible de réaliser une tomographie à partir de sources passives, comme le bruit de fond sismique, en utilisant un traitement mathématique (cross-corrélation, déconvolution; Pilz et al., 2013). Ainsi, la réponse sismique est déduite d'une station qui aurait bénéficié d'une source active pour une autre station. Cette approche a été utilisée avec succès dans le bassin de Barcelonnette dans des contextes de grands glissements profonds en contexte argileux ou en Asie centrale pour identifier des formations favorables aux glissements de terrain (Pilz et al., 2013). Les résultats des épaisseurs de formations identifiées sont en adéquation avec des résultats obtenus par des méthodes plus conventionnelles. 

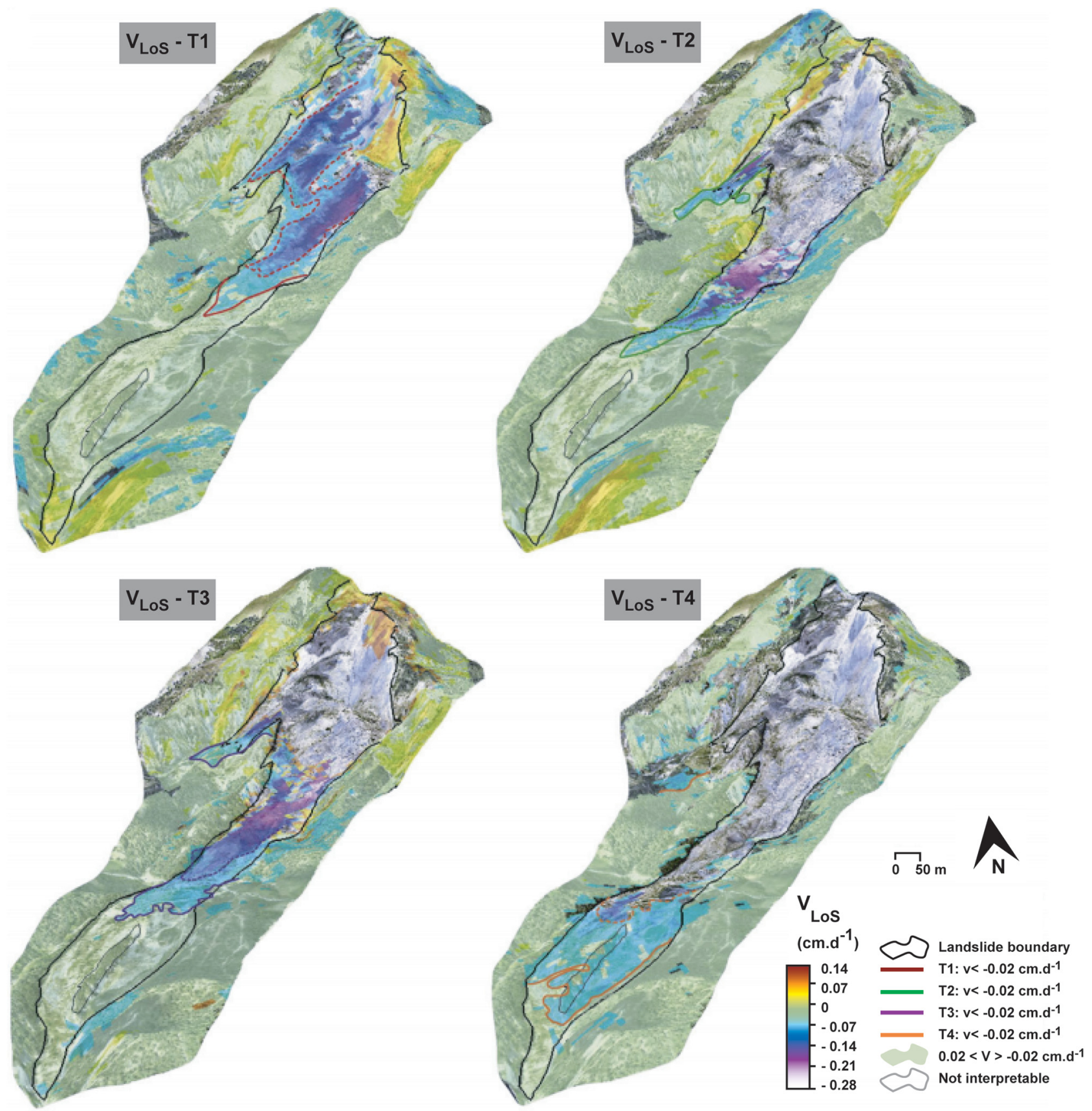

Fig. 5. Exemple de vitesses de déplacements calculées pour le glissement de la Valette à partir d'analyse d'images ALOS/PALSAR (d'après Schlögel et al., 2015).

Fig. 5. Example of computed velocities based on ALOS/PALSAR images analysis for La Valette earthflow (from Schlögel et al., 2015).

\subsubsection{Détection sismique automatique}

Il est possible localement, lorsqu'un réseau sismique est déployé, de détecter la localisation d'un phénomène et sa période de déclenchement(Bessason et al., 2007). Récemment, grâce à la densification du réseau sismique large bande à diverse échelles (mondiale, régionale, locale) il est possible de développer des approches de détection sismique automatique pour construire des catalogues de glissements de terrains (Hibert et al., 2017). Par exemple, Hibert et al. (2017) présentent une approche fondée sur une détection spectrale des signaux sismiques et l'identification des sources avec un algorithme de type « random forest $»$. La méthode, testée sur deux sites, en Himalaya et en Alaska, permet d'identifier (spatialement et temporellement) environ $80 \%$ des phénomènes identifiés géomorphologiquement par des techniques plus classiques. 

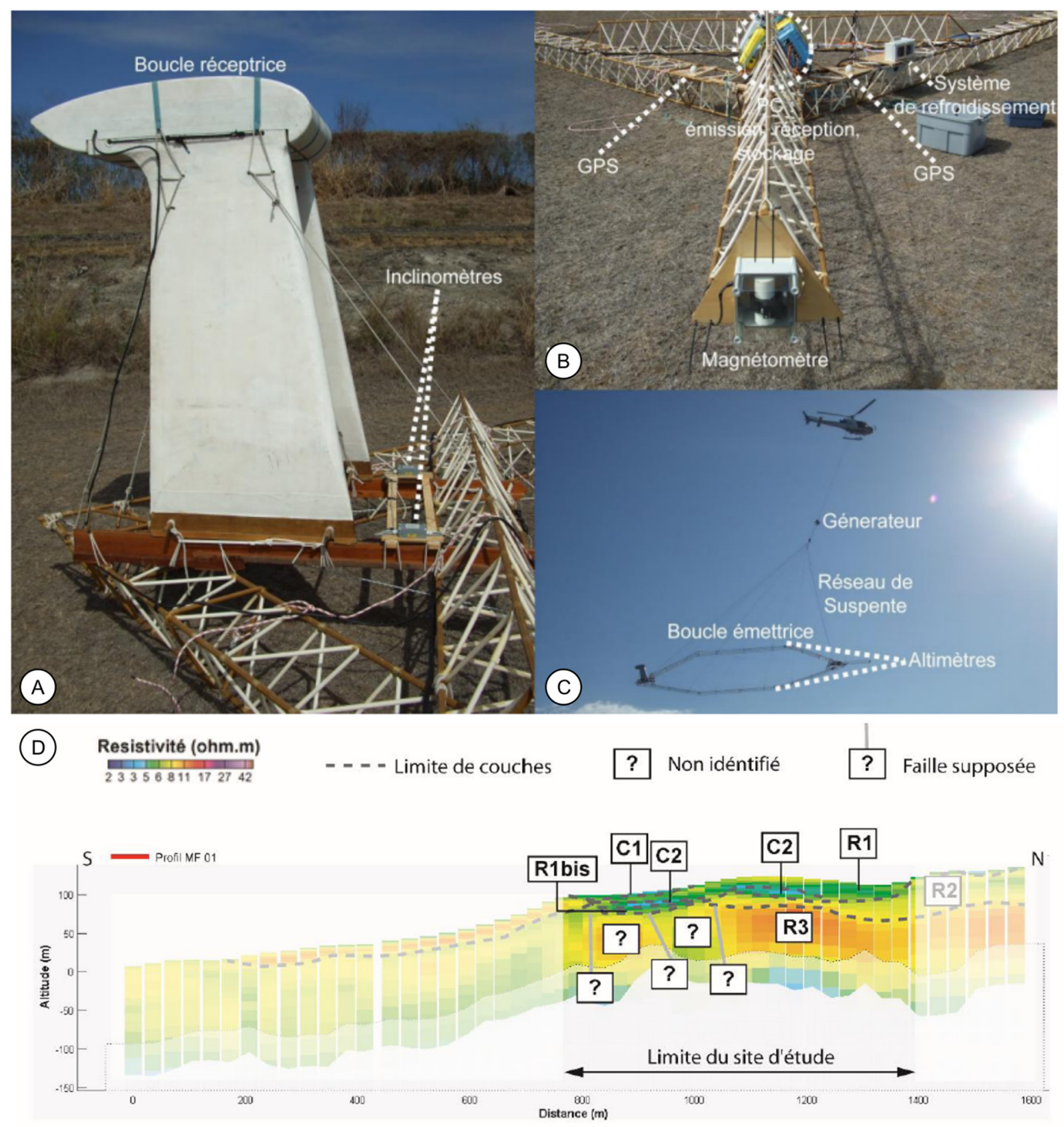

Fig. 6. Dispositif TDEM héliporté $\left(\right.$ SkyTEM $\left.^{\circledR}\right)$ utilisé par le BRGM pour les DROM (Reninger, 2012). A. Arrière du dispositif et de la boucle émettrice. B. Avant du dispositif et de la boucle émettrice. C. Dispositif en plein vol. D. Exemple de coupe de résistivité et interprétations (adapté de Thiery et al., 2016).

Fig. 6. Heliborne TDEM system (SkyTEM) used for DROMs by the French Geological Survey (Reninger, 2012). A. Back of the system with the transmitter loop. B. Front of the system with the transmitter loop. C. System in flight. D. Example of resistivity cross section and interpretations (adapted from Thiery et al., 2016).

\subsection{Nouveaux outils pour une cartographie de terrain}

La cartographie de terrain est une étape indispensable pour la cartographie de l'aléa glissements de terrain (Antoine et al., 2000) mais est très consommatrice de temps. Les outils numériques de saisie d'information spatiale sur le terrain permettent d'améliorer la cartographie et la localisation des phénomènes sur le terrain tout en diminuant le temps imparti à cette étape.
La manière de cartographier les glissements et la cartographie géomorphologique ont été révolutionnées par l'utilisation du GPS, permettant une localisation rapide des caractéristiques du paysage et des glissements de terrain avec parfois des précisions dépassant les besoins de l'expert (Malamud et al., 2004). Les outils de cartographie in situ, comme les tablettes avec SIG intégré, facilitent également la cartographie permettant d'améliorer les premières cartes 

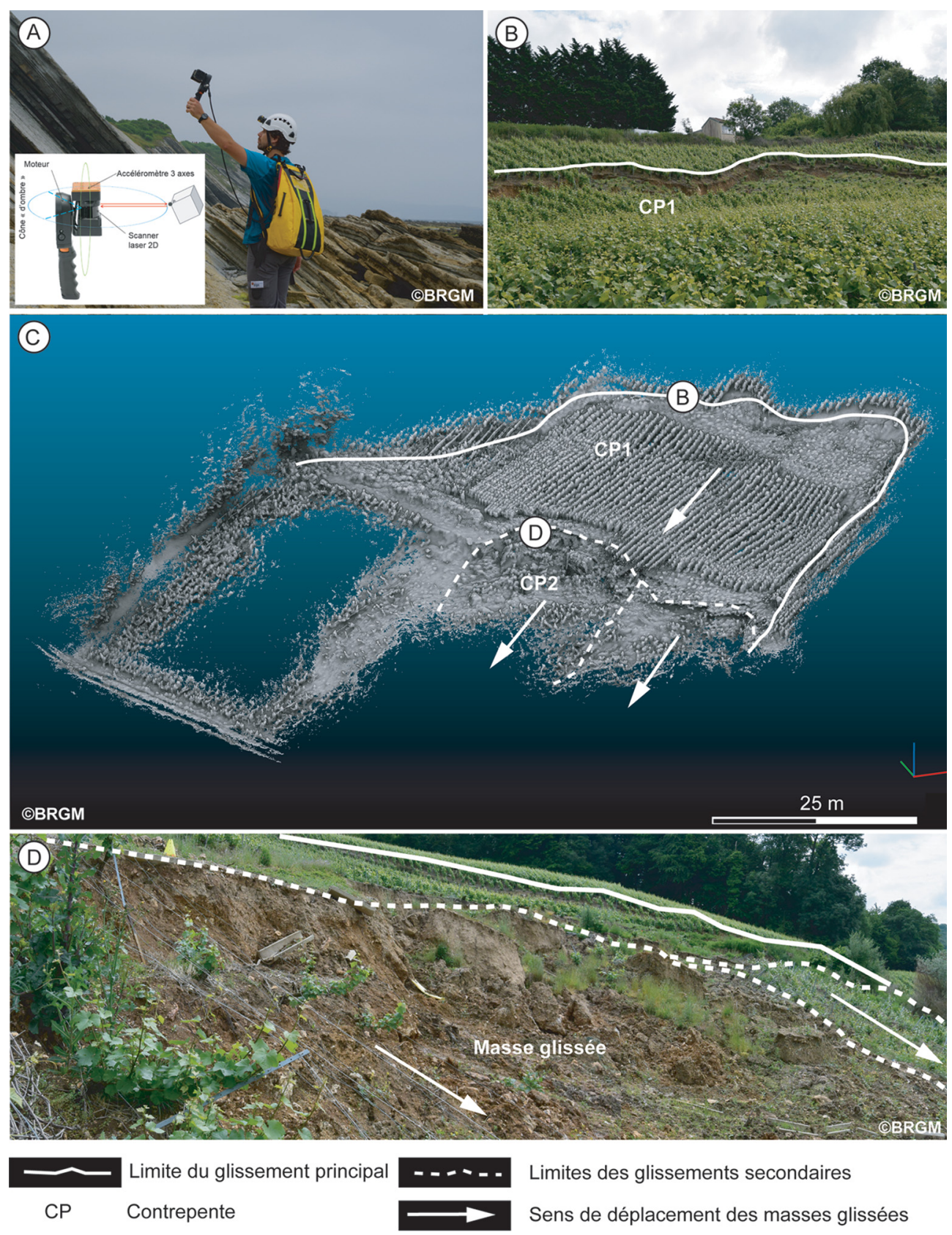

Fig. 7. Exemple de nuage de points obtenu par ZEB-Revo (scan laser terrestre 3D rotatif léger) pour un glissement de terrain de type rotationnel dans les vignes de Champagne (Commune de Le Mesnil-le Huttier-2018). A. Principes de fonctionnement du Zeb-Revo (lever sur la falaise de Socoa-Pyrénées Atlantique, France). B. Vue sur l'escarpement principal du glissement de terrain. C. Nuage de points et interprétation. D. Vue sur les glissements rotationnels secondaires.

Fig. 7. Example of a point cloud obtained with a ZEB-Revo (light rotating $3 D$ terrestrial laser scan) for a rotational landslide in the vineyards of Champagne (Commune of Le Mesnil-le Huttier-2018). A. Zeb-revo principles (measurements on the Socoa cliffs, Pyrénées Atlantique, France). $B$. View of the main landslide scarp. C. Point cloud and interpretation. D. View of the second landslide scarp.

réalisées par télédétection et d'ajouter directement sur le terrain des informations non vectorisées initialement. Récemment des essais de cartographies couplant jumelles électroniques («rangefinder binocular») connectées à une tablette et un GPS ont permis de lever à distance une série de glissements de terrain déclenchés par des précipitations pluvieuses (Santangelo et al., 2010). Une comparaison entre deux cartes réalisées, l'une sur le terrain à l'aide d'un GPS, l'autre par simple observation, a montré que les résultats étaient similaires, mettant alors en avant tout le potentiel de cette technologie nouvelle. Le Zeb-revo (laser rotatif léger, GeoSLAM $^{B}$ ), largement utilisé pour le zonage des cavités souterraines (Dewez et al., 2017), pourrait devenir également un outil de cartographie de précision des glissements de terrain. Des tests actuellement sont en cours sur des sites simples d'accès (Fig. 7), l'idée étant ensuite de le tester sur des 
sites plus complexes. Enfin, pour préciser et affiner des cartes de degré d'activité, le développement d'outils de prise d'images de haute qualité, de mesures in situ de déplacements et de déformation de surface par appareil low-coast (e.g. tagRFID) émergent et sont actuellement testés sur divers sites (Larosse, 2018).

\subsection{Des bases de données disponibles}

En France, les bases de données principalement utilisées pour la cartographie de type PPR sont disponibles sur le site Georisques maintenu par le Ministère de la Transition Ecologique et Solidaire. Les deux bases principalement concernées sont la BDMvt et la BSS. La BDMvt (base de données nationale des mouvements de terrain) recense tous les types de mouvements de terrain dont les glissements pour la métropole et les DROM (départements et régions d'outremer). C'est une base indispensable pour l'élaboration d'une carte d'aléa établie dans le cadre de PPR. Elle indique le type de mouvement de terrain, la précision planimétrique de la localisation, la date de déclenchement lorsqu'elle est disponible, le volume déstabilisé. La BSS (base de données du sous-sol) recense tous les forages de plus de $10 \mathrm{~m}$ de profondeur réalisés sur le territoire (DROM compris). Cette base est un complément non négligeable car des descriptions et des illustrations de forages sont souvent disponibles offrant plusieurs informations précises pour analyser les ruptures (types et épaisseur des formations, caractéristiques géotechniques des matériaux, profondeur de la nappe d'eau...).

\section{Perspectives pour le zonage d'aléa réglementaire ou que retenir pour les années à venir ?}

Les deux parties précédentes ont permis de dresser un état des lieux des techniques d'évaluation et de cartographie de l'aléa glissement de terrain ainsi que les évolutions récentes qui peuvent permettre une amélioration de la connaissance des phénomènes et de leur environnement. Si ces approches et techniques ont été testées avec succès dans le cadre de projets de recherche à différentes échelles, qu'en est-il pour une cartographie réglementaire? En d'autres termes, peut-on les utiliser pour évaluer l'aléa glissement de terrain dans le cadre d'un PPR? Et, dans ce cadre, quelles en seraient les limites?

\section{1 Évaluer les données disponibles pour améliorer l'inventaire}

Les techniques émergentes décrites précédemment permettent dans la majorité des cas de produire des données locales pour des projets spécifiques (recherche, etc.). Actuellement, il est difficilement envisageable dans une procédure PPR de les mettre en œuvre vu les moyens requis (connaissances géologiques et géotechniques initiales, temps de mise en œuvre et de mesure, précision, difficulté d'application sur un bassin de risque), sauf cas exceptionnel de révision locale préconisée par le guide PPR (MATE/MATL, 1999).
Pour les données altimétriques, il existe, en France, des données altimétriques gratuites de précision pour les missions de service public. Le tableau 4 présente différents types de données altimétriques nationales et internationales qui peuvent contribuer à une cartographie d'aléa pour un bassin de risque, notamment pour améliorer les inventaires des phénomènes (visuellement ou automatiquement) ou dériver des indices géomorphologiques contribuant au zonage. Toutefois, malgré les recommandations qualitatives indiquées dans ce tableau, il convient de faire une analyse des données avant de démarrer l'étude (analyse visuelle ou par calcul de dérivées premières et secondes de type pente, exposition ou courbure). En effet, pour certaines localisations, certains MNT, comme le RGAlti $5 \mathrm{~m}$ ou RGEAlti $1 \mathrm{~m}$ sont donnés avec 1 ou $5 \mathrm{~m}$ de précision en planimétrie et $1 \mathrm{~m}$ de précision en altimétrie. Certains sont issus d'un assemblage de différentes données hétérogènes qui sont ré-échantillonnées avec localement des secteurs qui n'apportent pas d'information supplémentaire (Fig. 8).

Si les images satellites permettent une cartographie rapide en cas de crise (Stumpf et Kerle, 2011), leur utilisation semble complexe dans le cadre de PPR, notamment les images radar qui demandent des temps de traitement longs ou complexes (Tab. 4). Il semble plus concret d'utiliser des ortho-images issues de photographies aériennes dont les résolutions peuvent être d'ordre métrique (Tab. 4). L'IGN met à disposition gratuitement ce type de données (BD ortho $5 \mathrm{~m}$ ). Des photographies aériennes ou des images satellites de résolution infra métrique existent, par exemple la BD ortho $50 \mathrm{~cm}$. En effet, des outils sur le traitement semi-automatique et automatique par classification et analyse des textures et de la structure des objets (outils comme eCognition ${ }^{\circledR}$ ou la suite ENVI $)$ existent, mais ont une utilisation complexe pour des résultats pas toujours satisfaisants (Moine et al., 2009). À ce jour, l'analyse visuelle sera privilégiée.

Les données géophysiques sont acquises localement pour des situations spécifiques. Aussi, il n'est pas envisageable de les utiliser pour l'élaboration de PPR sauf dans le cadre de révisions de PPR où l'acquisition de données supplémentaires est nécessaire pour améliorer le zonage (MATE/MATL, 1999).

Enfin, les bases de données disponibles telle que la BSS ou la BDMvt fournissent localement des informations sur le sol, le sous-sol et les phénomènes. Ces dernières sont à consulter impérativement dans le cadre du zonage d'aléa PPR (Tab. 4) avec comme le souligne Fressard (2013) une analyse critique à réaliser sur la typologie, la précision des localisations des phénomènes et les dates de déclenchement.

\subsection{Quelle approche spatiale numérique pour un zonage d'aléa réglementaire?}

Le tableau 5 indique de manière théorique les méthodes qui peuvent être utilisées dans un cadre réglementaire tel que les PPR. En France, ces approches spatiales d'évaluation de l'aléa glissements de terrain sont surtout utilisées dans le cadre de la recherche. Des essais de comparaisons entre cartes d'aléa produites par méthodes qualitatives (méthodes directes et indirectes) et produites par méthodes quantitatives (méthodes bivariées, multivariées, et déterministes) à l'échelle du 
Tableau 4. Aperçu de données disponibles (liste non exhaustive) et de leur utilisation pour une cartographie d'aléa glissements de terrain et applicabilité dans le cadre d'un PPR.

Table 4. Overview of available data (non-exhaustive list) and their use for landslide hazard mapping and applicability in the framework of a PPR.

\begin{tabular}{|c|c|c|c|c|c|c|c|}
\hline \multirow[t]{2}{*}{ Thème } & \multicolumn{2}{|l|}{ Données à utiliser } & \multirow[t]{2}{*}{ Disponibilité } & \multirow[t]{2}{*}{$\begin{array}{l}\text { Organisme chargé } \\
\text { de la donnée }\end{array}$} & \multirow[t]{2}{*}{$\begin{array}{l}\text { Domaine } \\
\text { d'utilisation }\end{array}$} & \multicolumn{2}{|c|}{$\begin{array}{c}\text { Applicabilité } \\
\text { pour le zonage PPR }\end{array}$} \\
\hline & Type & Produit & & & & Importance & Précautions \\
\hline \multirow{14}{*}{$\begin{array}{l}\text { Inventaire des } \\
\text { glissements } \\
\text { de terrain et } \\
\text { géomorphologie }\end{array}$} & $\begin{array}{l}\text { Base de données } \\
\text { glissements }\end{array}$ & BDMvt & $\mathrm{TU}$ & MTES/BRGM & $\mathrm{I} / \mathrm{S} / \mathrm{A}$ & +++ & $(\mathrm{X})$ \\
\hline & Cartes topographiques & SCAN $25^{\circledR}$ & & IGN & $\mathrm{I} / \mathrm{S} / \mathrm{A}$ & +++ & \\
\hline & Orthophotographies & BDOrtho $5 \mathrm{~m}$ & $\mathrm{TU}$ & IGN & $\mathrm{I} / \mathrm{S} / \mathrm{A}$ & +++ & \\
\hline & & BDOtho & MSP & & & ++ & \\
\hline & & $50 \mathrm{~cm}$ & MSP & & & + & \\
\hline & & BDOrtho $^{\infty}$ & ERE & & & ++ & \\
\hline & & & $\mathrm{TU}$ & & & +++ & \\
\hline & & BDOrtho & & & & & \\
\hline & & $\begin{array}{l}\text { historique } \\
\text { Remonter le temps }\end{array}$ & & & & & \\
\hline & Images satellites & Pleiades & ERE & IGN & $\mathrm{I} / \mathrm{S} / \mathrm{A}$ & + & \\
\hline & & Images SPOT & ERE & IGN & & + & \\
\hline & & Ortho HR & ERE & IGN & & + & \\
\hline & & & $\mathrm{TU}$ & ESA/Copernicus & & & \\
\hline & & Sentinel & & & & & \\
\hline \multirow{9}{*}{$\begin{array}{l}\text { Topographie } \\
\text { et dérivées }\end{array}$} & Base de données & BD Alti $250 \mathrm{~m}$ & $\mathrm{TU}$ & IGN & $\mathrm{I} / \mathrm{S} / \mathrm{A}$ & - & $(\mathrm{XX})$ \\
\hline & altimétriques/MNT & BD Alti & $\mathrm{TU}$ & IGN & & - & $(\mathrm{XX})$ \\
\hline & & $75 \mathrm{~m}$ & MSP & IGN & & ++ & $(\mathrm{XX})$ \\
\hline & & BD Alti $25 \mathrm{~m}$ & MSP & IGN & & +++ & (X) \\
\hline & & RGE Alti & MSP & IGN & & +++ & $(\mathrm{X})$ \\
\hline & & $5 \mathrm{~m}$ & $\mathrm{TU}$ & MTI/NASA & & + & \\
\hline & & RGE Alti $1 \mathrm{~m}$ & $\mathrm{P}$ & & & + & $(\mathrm{XX})$ \\
\hline & & ASTER & ou SD & DLR/EADSAstrium & & & (X) \\
\hline & & WorlDEM & & & & & \\
\hline \multirow[t]{4}{*}{ Géologie et sol } & Cartes géologiques & Papier & $\mathrm{P}$ & BRGM & $\mathrm{I} / \mathrm{S} / \mathrm{A}$ & +++ & $(\mathrm{XX})$ \\
\hline & Cartes du & vectorisées & $\mathrm{P}$ & BRGM & $\mathrm{I} / \mathrm{S} / \mathrm{A}$ & + & $(\mathrm{XX})$ \\
\hline & régolithe & & ND & BRGM & $\mathrm{S}$ & - & $(\mathrm{XX})$ \\
\hline & Carte des types de sols & & SD & INRA/IRD & S/A & ++ & $(\mathrm{XX})$ \\
\hline \multirow[t]{3}{*}{ Hydrologie } & Carte du réseau & BD Carthage & $\mathrm{TU}$ & IGN & S & + & $(\mathrm{XX})$ \\
\hline & hydrographique & & SBV & Service de l'État & $\mathrm{S} / \mathrm{A}$ & + & \\
\hline & Profondeur de nappe & & & & & & \\
\hline Occupation du sol & Cartes de l'occupation du sol & CorineLandCover & $\mathrm{TU}$ & MTES & $\mathrm{S}$ & + & \\
\hline Climat & Données précipitations & & SA & MétéoFrance & $\mathrm{S} / \mathrm{A}$ & + & $(\mathrm{X})$ \\
\hline \multirow{2}{*}{$\begin{array}{l}\text { Séisme/accélération } \\
\text { sismique }\end{array}$} & Données sismiques & & $\mathrm{TU}$ & BCSF/RéNaSS & $\mathrm{S} / \mathrm{A}$ & + & \\
\hline & et localisation & & $\mathrm{TU}$ & Sisfrance & & & \\
\hline \multirow[t]{2}{*}{ Applications web } & Georisques (BDMvt/BSS/cartes & Georisque & TU & MTES/BRGM & $\mathrm{I} / \mathrm{S} / \mathrm{A}$ & ++ & $(\mathrm{X})$ \\
\hline & géologiques) & Google earth & $\mathrm{TU}$ & Google earth & $\mathrm{I} / \mathrm{S} / \mathrm{A}$ & ++ & \\
\hline
\end{tabular}

Note: $\mathrm{TU}=$ disponible pour tout usage $; \mathrm{MSP}=$ disponible pour mission d'utilité publique ; ERE= disponible pour l'État et organisme de recherche; $\mathrm{P}=$ payant $; \mathrm{SD}=$ sur demande; $\mathrm{SA}=$ disponible selon abonnement $;+++=$ indispensable $;++=$ important $;+=$ moyennement important $;-=$ peu important $;(\mathrm{X})=$ précaution à prendre (vérification des données) $;(\mathrm{XX})=$ vérification de l'échelle d'application à réaliser ; $\mathrm{I}=$ inventaire $; \mathrm{S}=$ susceptibilité $; \mathrm{A}=$ aléa.

$T U=$ available for any use MSP = available for public utility mission; ERE= available for the State and research organism; $P=$ paying service; $S D=$ on request; $S A=$ available by subscription; $+++=$ essential; $++=$ important; $+=$ moderately important; $-=$ not very important; $(X)=$ precaution (data verification); $(X X)=$ verification of the scale of application; $I=$ inventory; $S=$ susceptibility; $A=$ hazard. 


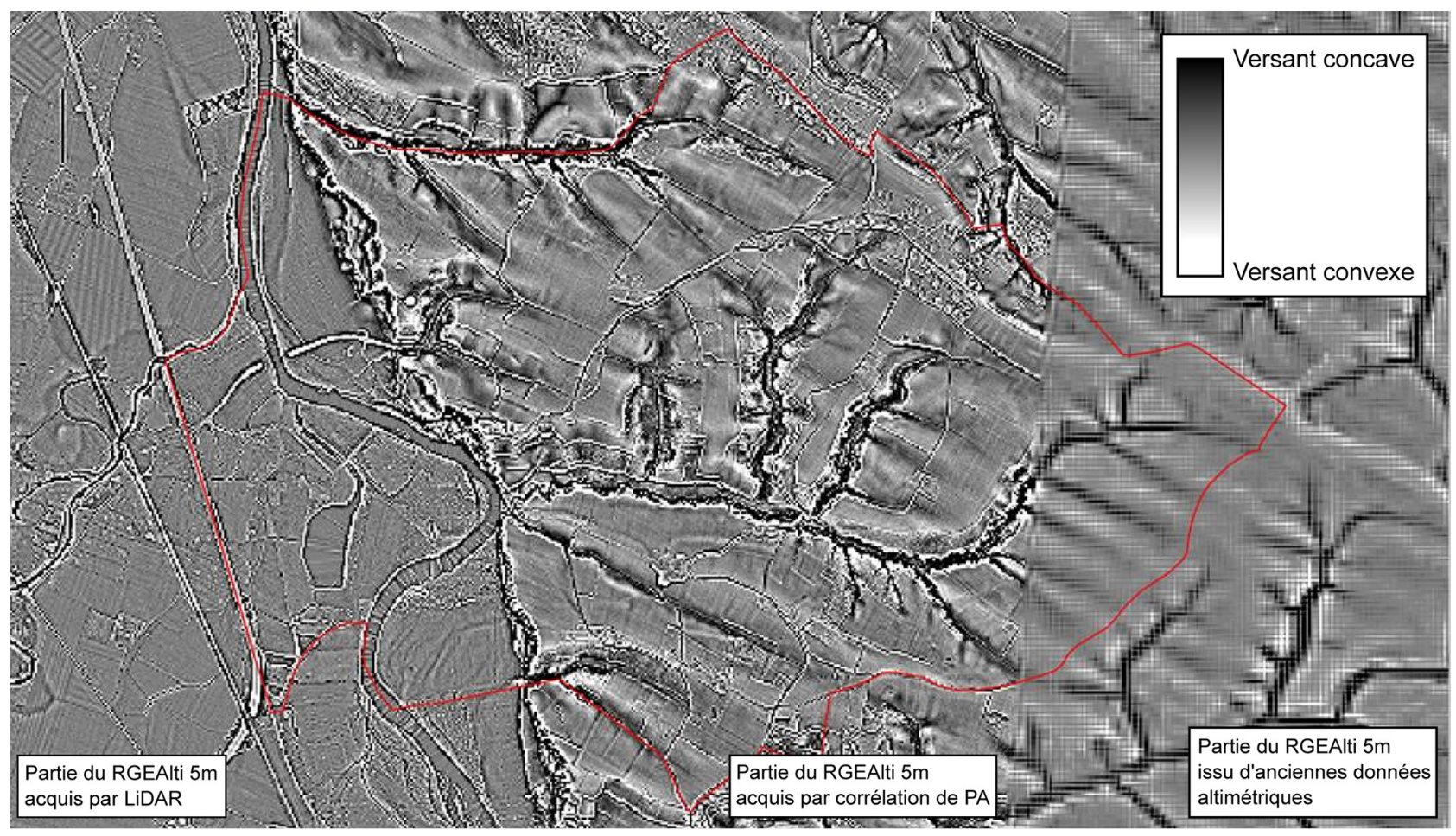

Fig. 8. Exemple de problèmes liés au RGEAlti $5 \mathrm{~m}$ issus d'un assemblage de plusieurs techniques et données altimétriques hétérogènes sur la commune de Clermont-le-Fort (31). La carte représente une dérivée (le degré de courbure représentée en nuances de gris) issue du même MNT. Fig. 8. Example of problems related to the DTM RGEAlti $5 \mathrm{~m}$ resulting from a merge elevation data from several techniques and heterogeneous database (location: municipality of Clermont-le-Fort-31). The map represents a derivative (curvature is represented by grey shades) from the same DTM.

Tableau 5. Synthèse des différents types et niveaux de complexité de zonage selon les objectifs cartographiques (modifié de Fell et al., 2008). Table 5. Summary of different types, levels and complexity of landslide susceptibility and hazard zoning according to the objectives (modified from Fell et al., 2008).

\begin{tabular}{|c|c|c|c|c|c|c|c|c|}
\hline \multirow[t]{2}{*}{ Objectifs } & & \multicolumn{3}{|c|}{ Type de zonage } & \multicolumn{3}{|c|}{ Niveau de précision du zonage } & \multirow[t]{2}{*}{ Échelle des cartes } \\
\hline & & Inventaire & Susceptibilité & Aléa & Basique & Intermédiaire & Sophistiqué & \\
\hline \multirow[b]{2}{*}{ Zonage régional } & Informatif & $\mathrm{X}$ & $X$ & $(\mathrm{XX})$ & $\mathrm{X}$ & $(\mathrm{X})$ & NA & \multirow[t]{2}{*}{$1: 250000-1: 25000$} \\
\hline & Réglementaire & {$[\mathrm{X}]$} & NA & NA & NA & NA & NA & \\
\hline \multirow{2}{*}{ Zonage local } & Informatif & $\mathrm{X}$ & $\mathrm{X}$ & $\mathrm{X}$ & $\mathrm{X}$ & $(\mathrm{X})$ & $(\mathrm{XX})$ & \multirow[t]{2}{*}{$1: 25000-1: 5000$} \\
\hline & Révision & $(\mathrm{X})$ & $(\mathrm{X})$ & $(\mathrm{XX})$ & $X$ & $(\mathrm{XX})$ & NA & \\
\hline \multirow{3}{*}{ Site } & Informatif & {$[\mathrm{X}]$} & & & & & $\mathrm{X}$ & \multirow[t]{3}{*}{$>1: 5000$} \\
\hline & Consultatif & $(\mathrm{X})$ & & & & $\mathrm{X}$ & $\mathrm{X}$ & \\
\hline & Réglementaire & $(\mathrm{X})$ & $X$ & & & & & \\
\hline
\end{tabular}

Note: $\mathrm{X}=$ applicable $;(\mathrm{X})=$ applicable avec quelques précautions (e.g. analyse des données d'entrée); $(\mathrm{XX})=$ développements spécifiques nécessaires ; $[\mathrm{X}]=$ non recommandé ; NA = non adapté ; en gras domaine d'application pour cartographie d'aléa réglementaire.

$X=$ applicable; $(X)=$ applicable with some precautions (e.g. analysis of input data); $(X X)=$ specific developments required; $[X]=$ not recommended; $N A=$ not suitable; in bold = field of application for regulatory hazard mapping. 
$1: 10000^{\text {ème }}$ ont été réalisés, notamment dans le bassin de Barcelonnette (Thiery, 2007; Thiery et al., 2007, 2017a). Si les résultats des méthodes quantitatives sont prometteurs, ils révèlent également quatre problèmes majeurs :

- le premier problème est lié au calage des modèles qui peut s'avérer long selon l'approche retenue (Thiery, 2007; Thiery et al., 2014). En effet, selon la qualité des données d'entrée, des problèmes mathématiques (problème de dépendance conditionnelle, fortes incertitudes) peuvent biaiser les résultats, surestimant ou sous-estimant les probabilités finales (Thiery et al., 2005). De plus, il est nécessaire de créer des modèles par type de glissements de terrain, chaque type ayant ses facteurs de prédisposition propres (Thiery, 2007);

- le deuxième problème est lié aux variables qui sont utilisées. S'il a été prouvé que les pondérations peuvent être transposées d'un site à un autre si le contexte géomorphologique est similaire (Thiery, 2007), il faut que les données d'entrée soient exactement les mêmes (en terme de résolution et de classes de variables), ce qui est rarement le cas d'un bassin de risque à un autre;

- le troisième problème est lié aux classes d'aléa retenues pour les cartes modélisées. En principe des classes d'aléa sont obtenues par discrétisation des courbes de probabilités cumulées calculées. Plusieurs approches existent (seuils naturels, moyennes emboitées, quantiles, méthode de Jenks, etc.). Toutefois, aucun consensus actuellement n'est établi sur la méthode à utiliser, ce qui pose un souci d'homogénéisation entre cartes réalisées par des experts différents et ayant une sensibilité différente.

- enfin, les développements numériques récents (intelligence artificielle, méthodes issues du data mining) tendent à augmenter le nombre d'approches, qui apparaissent comme étant toutes efficaces avec de forts taux de reconnaissance des évènements. Toutefois, ces approches apparaissent parfois très complexes. Dans ce cas, retranscrire de manière simple les étapes de l'évaluation de l'aléa aux acteurs locaux peut se révéler périlleux voire impossible.

Au regard de ce constat, il apparaît difficile d'appliquer, dans un contexte réglementaire, ces types de méthodes. Il est préférable de se focaliser sur les méthodes qualitatives dites indirectes comme les logiques floues ou l'AHP (processus d'analyse hiérarchique; Saaty, 1977) ou encore des approches hybrides mixant méthodes qualitatives indirectes et méthodes statistiques/probabilistes (Thiery et al., 2014). Ces techniques, développées pour analyser des décisions complexes, permettent des prises de décisions en confrontant des groupes de personnes (experts, décideurs) autour d'un problème et de sa compréhension. Outre le fait que ces techniques permettent de créer des scenarii et de confronter des hypothèses rapidement, elles introduisent la vision experte dans le processus de cartographie ce qui permet d'intégrer des changements subtils que les approches quantitatives ne permettent pas (Thiery, 2007). Testées depuis une dizaine d'années pour évaluer l'aléa glissements de terrain à petite échelle et de plus en plus utilisées à échelle locale $\left(1: 10000^{\text {ème }}\right)$, c'est à notre sens les techniques les plus adaptées actuellement pour l'évaluation dans un cadre réglementaire (Thiery et al., 2014).

\subsection{Stratégie pour la révision locale des PPR}

Inversement, pour des révisions de PPR, des techniques de zonage d'aléa sophistiquées sont envisagées voire conseillées. Dans ces cas particuliers, comme évoqué précédemment, l'acquisition de données supplémentaires pour améliorer le zonage sont préconisées par le guide PPR (existence d'enjeux majeurs humains ou socio-économiques et d'intérêt public). C'est ce qui a été réalisé récemment en Martinique à la demande la commune de la Trinité. Le projet vise à aménager un secteur agricole cartographié en aléa modéré mais susceptible d'absorber une partie de la forte croissance démographique de cette commune. Les DROM ayant bénéficié d'une couverture TDEM héliportée complète (projet TDEM héliporté BRGM/Sky$\mathrm{TEM}^{\mathbb{8}}$ ), il a été proposé une étude multidisciplinaire mixant intégration de données issues de ces levés pour identifier les épaisseurs d'altération, création d'un modèle 3D du secteur, modélisation physique par profils caractéristiques sous TALREN $^{\circledR}$ (Terrasol, 2018) des effets de l'aménagement sur les versants, modélisation spatiale à base physique $2,5 \mathrm{D}$ d'équilibre limite $\left(\right.$ ALICE $\left.^{B}\right)$ et de la propagation $\left(\right.$ BORA $\left.^{\circledR}\right)$ des phénomènes superficiels et profonds(Fig. 9, Thiery et al., 2017b). Les résultats ont montré que le secteur cartographié en aléa modéré (susceptibilité en réalité) pouvait comporter des secteurs soumis à un aléa fort de glissements de terrain. Un secteur apparaissait en aléa nul à faible et pouvait potentiellement accueillir un projet d'aménagement. Ce projet montre également qu'il est possible localement d'intégrer numériquement la propagation des matériaux déstabilisés dans le zonage d'aléa type PPR.

\section{Conclusion}

Dès lors qu'un zonage d'aléa glissements de terrain doit être établi, en préalable au cahier des charges, quatre questions doivent être posées relatives : (i) à son objectif (pour quel usage ?); (ii) au type d'information recherchée; (iii) au niveau ou degré de précision; (iv) à l'échelle cartographique attendue. Le tableau 5 présente des indications sur la qualité du zonage nécessaire en fonction des objectifs dans le cadre d'une étude cartographique de l'aléa glissements de terrain. Ainsi, associé au tableau 4, relatif aux caractéristiques des données de base disponibles, ce tableau 5 peut permettre d'orienter le choix des méthodes à mettre en œuvre en fonction du contexte, des données disponibles et des objectifs visés pour la prévention des risques naturels.

Pour conclure, le zonage d'aléa réglementaire en France est parfois controversé du fait de la méthodologie subjective préconisée. Comme le souligne le guide PPR mouvements de terrain «ce type d'analyse est privilégié par rapport aux études spécifiques qui débordent du cadre du PPR et de la mission de l'État» (MATE/MATL, 1999). Pourtant, sans s'affranchir de l'opinion experte, par l'utilisation de nouvelles données et de nouvelles approches, il est possible de préciser l'inventaire, de formaliser certaines règles cartographiques et ainsi d'améliorer la cartographie d'aléa glissements de terrain. Au regard de ces considérations (nouvelles données disponibles avec une résolution spatiale fine, nouveaux outils de traitement disponibles, évolution du contexte naturel) il conviendrait, dans certains cas, de remettre à jour certains PPR, de les repréciser, et au-delà envisager de modifier le guide PPR pour tenir compte de ces évolutions techniques et technologiques. 


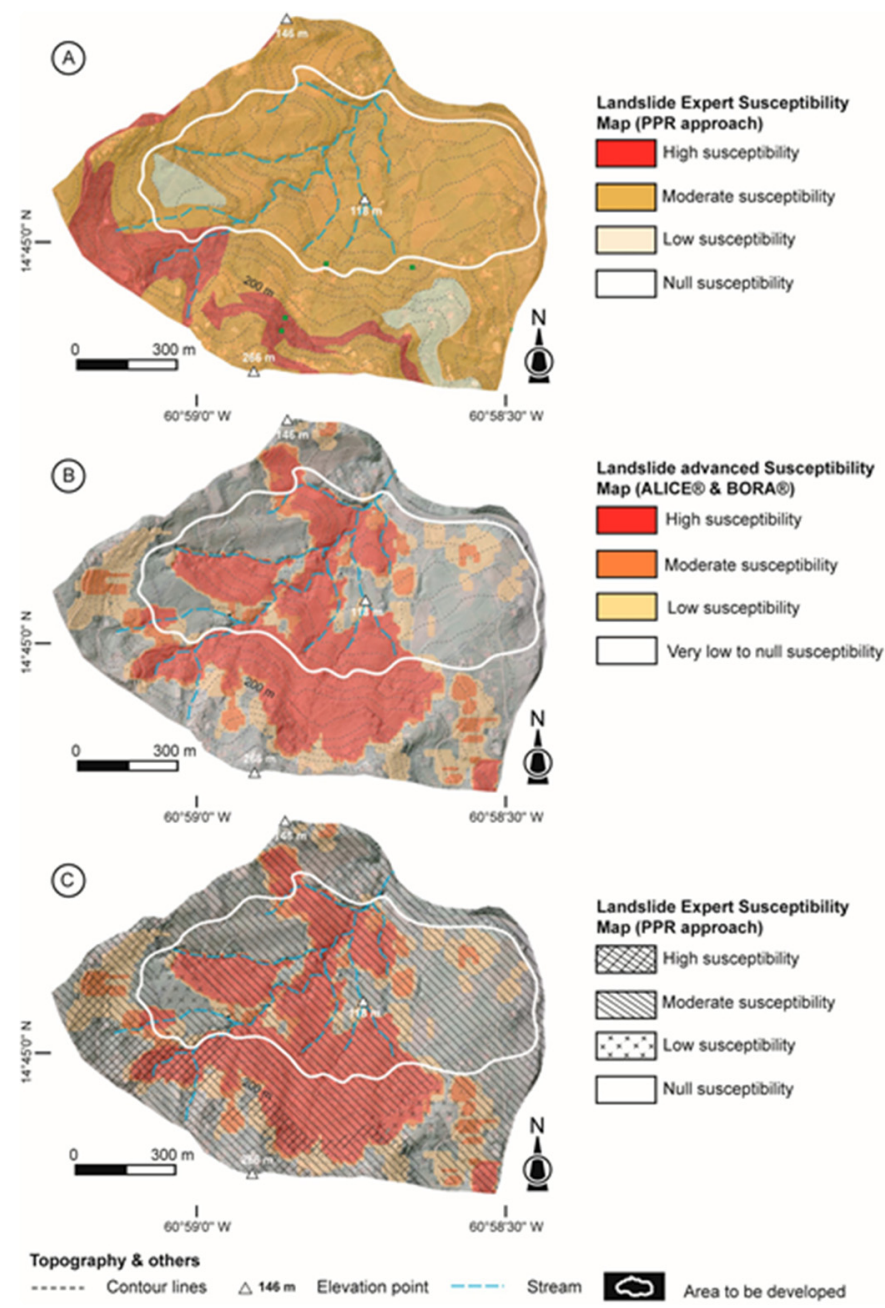

Fig. 9. Exemple de carte de susceptibilité avancée glissements de terrain pour la commune la Trinité (Martinique, d'après Thiery et al., 2017b). A. Carte produite par approche empirique pour le PPR de la commune de la Trinité. B. Carte produite par approche multidisciplinaire. C. Superposition des deux cartes (A, B).

Fig. 9. Example of an "advanced" susceptibility map for the commune of La Trinité (Martinique, from Thiery et al., 2017b). A. landslide hazard map produced by empirical approach for the PPR of municipality of La Trinité. B. Map produced using a multidisciplinary approach. C. Overlay of the two maps $(A, B)$.

Remerciement. Cette recherche a été soutenue financièrement par la DGPR, contrat MEZAG-AP19DRP018 et le BRGM, contrat LANDMASS-RP17DRP030. Le manuscrit a grandement bénéficié des commentaires constructifs de l'éditeur et des correcteurs anonymes.

\section{Références}

Aleotti P, Chowdhury R. 1999. Landslide hazard assessment: Summary review and new perspectives. Bull Eng Geol Environ 58: 21-44.

Antoine P, Debelmas J, Durville JL. 2010. Aux origines de la réglementation française actuelle en matière de mouvements de versants : la coulée du plateau d'Assy en 1970. Rev Fr Geotech 131-132: 71-80.

Antoine P, Cojean R, Durville JL et al. 2000. Caractérisation et cartographie de l'aléa dû aux mouvements de terrain. Guide technique. Laboratoire Central des Ponts et Chaussées, Collection environnement, risques naturels, Paris, $91 \mathrm{p}$.

Bessason B, Eiriksson G, Thorarinsson O, Thorarinsson A, Einarsson S. 2007. Automatic detection of avalanches and debris flows by seismic methods. J Glaciol 53(182): 461-472.

BRGM. 1976. Zones exposées à des risques liés aux mouvements du sous-sol, carte Zermos: Trouville, Pont-l'Evêque (Calvados).

Cascini L. 2008. Applicability of landslide susceptibility and hazard zoning at different scales. Eng Geol 102: 164-177.

Champetier de Ribes G. 1987. La cartographie des mouvements de terrain, des ZERMOS aux PER. Bull Liaison Lab Ponts Chaussées $\mathrm{N}^{\mathrm{o}}$ Spécial 150/151: 9-19.

Chen W, Xie X, Wang J et al. 2016. A comparative study of logistic model tree, random forest, and classification and regression tree models for spatial prediction of landslide susceptibility. Catena 151: $147-160$

Chen W, Shirzadi A, Shahabi H et al. 2017. A novel hybrid artificial intelligence approach based on the rotation forest ensemble and naïve Bayes tree classifiers for a landslide susceptibility assessment in Langao County, China. Geomat Nat Hazard Risk 8(2): 1955-1977.

Coe JA, Michael JA, Crovelli RA, Savage WZ, Laprade WT, Nashem WD. 2004. Probabilistic assessment of precipitation-triggered landslides using historical records of landslide occurrence, Seattle, Washington. Environ Eng Geosci 10(2): 103-122.

Corominas J, van Westen C, Frattini P et al. 2014. Recommendations for the quantitative analysis of landslide risk. Bull Eng Geol Environ 73: 209-263.

Crovelli RA. 2000. Probability models for estimation of number and costs of landslides. U.S. Geological Survey, Open File Report 00-249, Denver.

Cruden DM, Fell R, (eds.). 1997. Landslide risk assessment. Proceedings of the International Workshop on Landslide Risk Assessment, Honolulu, Hawaï, USA, Balkema, 371 p.

Dang K, Sassa K, Fukuoka H et al. 2016. Mechanism of two rapid and long-runout landslides in the 16 April 2016 Kumamoto earthquake using a ring-shear apparatus and computer simulation (LSRAPID). Landslides 13(6): 1525-1534.

Dewez TJB, Thuon Y, Yart S, Plat E, Pannet P. 2017. Towards cavity collapse hazard maps with Zeb-Revo handheld laser scanner point clouds. Photogramm Rec, doi: 10.1111/phor.12223.

DREAL. 2004. Notice d'utilisation de la carte de prédisposition aux mouvements de terrain. Les documents de la DREAL, Normandie, $5 \mathrm{p}$. Disponible sur http://www.donnees.normandie.developpe ment-durable.gouv.fr/pdf/MVT/Notice_MVT.pdf.

Fell R, Ho KKS, Lacasse S, Leroi E. A framework for landslide risk assessment and management. In: Hungr O, Fell R, Couture R, Eberhardt E, (eds). Landslide risk management. London: Taylor and Francis, 2005, pp. 3-26.

Fell R, Corominas J, Bonnard C, Cascini L, Leroi E, Savage W. 2008. Guidelines for landslide susceptibility, hazard and risk zoning for land use planning. Eng Geol 102(3-4): 85-98.

Frappa M, Lebourg T. 2001. Mesures géophysiques pour l'analyse des glissements de terrain. Rev Fr Geotech 95-96: 33-39.

Fressard M. 2013. Les glissements de terrain du Pays d'Auge continental (Normandie, France). Caractérisation, cartographie, analyse spatiale et modélisation. Ph.D. Thesis, University of Caen Basse-Normandie, Caen.

Fressard M, Thiery Y, Maquaire O. 2014. Which data for quantitative landslide susceptibility mapping at operational scale? Case study of 
the Pays d'Auge plateau hillslopes (Normandy, France). Nat Hazard Earth Syst Sci 14: 569-588.

Grislain-Letrémy C, Peinturier C. 2010. Le régime d'assurance des catastrophes naturelles en France métropolitaine entre 1995 et 2006. Commissariat général du développement durable : service de l'économie, de l'évaluation et de l'intégration du développement durable, Rapport $\mathrm{n}^{\circ} 22$, MEEDDAT, La Défense.

Glade T, Crozier MJ. A review of scale dependency in landslide hazard and risk analysis. In: Glade T, Anderson M, Crozier MJ, (eds). Landslide hazard and risk. Chichester, UK: Wiley, 2005, pp. $75-138$.

Guillope P, Porcher M. 1979. Cartographie des risques ZERMOS appliquée à des plans d'occupation des sols en Normandie. Connaitre le sous-sol: un atout pour l'aménagement urbain, Colloque national, pp. 667-677.

Guzzetti F, Mondini AC, Cardinali M, Fiorucci F, Santangelo M, Chang KT. 2012. Landslide inventory maps: New tools for an old problem. Earth-Sci Rev 112(1): 42-66.

Hibert C, Malet JP, Provost F, Michéa D, Geertsema M. 2017. Automated seismic detection of landslides at regional scales: A Random Forest based detection algorithm for Alaska and the Himalaya. Geophysical Research Abstracts Vol. 19, EGU2017-6151, EGU General Assembly, Vienna, 2017.

Horton P, Jaboyedoff M, Rudaz B, Zimmermann M. 2013. Flow-R, a model for susceptibility mapping of debris flows and other gravitational hazards at a regional scale. Nat Hazard Earth Syst Sci 13: 869-885.

Humbert M. 1977. La cartographie en France des zones exposées à des risques liés aux mouvements du sol-Cartes zermos. Bull Int Assoc Eng Geol-Bull Assoc Int Geol Ing 16(1): 80-82.

Iwahashi J, Pike RJ. 2007. Automated classifications of topography from DEMs by an unsupervised nested means algorithm and a three-part geometric signature. Geomorphology 86(3-4): 409-440.

Jongmans D, Garambois S. 2007. Geophysical investigation of landslides: A review. Bull Soc geol Fr 178(2): 101-112.

Larosse E. 2018. L'utilisation des méthodes géophysiques dans le early warning system: Perspectives. Journées biennales des géosciences de l'UNIL-Session : Au-delà de l'aléa ou comment proposer une gestion intégrée des risques naturels, 15 février 2018 , Lausanne, Suisse.

Ledoux B. 1995. Les catastrophes naturelles en France. Documents Payot, Paris, $450 \mathrm{p}$.

Malamud BD, Turcotte DL, Guzzetti F, Reichenbach P. 2004. Landslide inventories and their statistical properties. Earth Surf Process Landf 29: 687-711.

Maquaire O. 2002. Aléas géomorphologiques (mouvements de terrain): processus, fonctionnement, cartographie. Mémoire d'habilitation à Diriger des Recherches, Strasbourg: Université Louis Pasteur.

MATE/MATL. 1997. Plan de prévention des risques (PPR) : plans de prévention des risques littoraux (PPR): guide méthodologique. Ministère de l'Aménagement du Territoire et de l'Environnement (MATE), Ministère de l'Équipement des Transports et du Logement (METL) . Paris : La Documentation Française.

MATE/MATL. 1999. Plan de prévention des risques (PPR) : risques de mouvements de terrain. Ministère de l'Aménagement du Territoire et de l'Environnement (MATE), Ministère de l'Équipement des Transports et du Logement (METL). Paris: La Documentation Française.

Mergili M, Marchesini I, Rossi M, Guzzetti F, Fellin W. 2014. Spatially distributed three-dimensional slope stability modelling in a raster GIS. Geomorphology 206: 178-195.
Moine M, Puissant A, Malet JP. Detection of landslides from aerial and satellite images with a semi-automatic method. Application to the Barcelonnette basin (Alpes-de-Haute-Provence, France). In: Malet JP, Remaitre A, Bogaard T, (eds). Landslide processes: From geomorphological mapping to dynamic modelling, Strasbourg, France: CERG, 2009, pp. 63-68.

Pastor M, Blanc T, Haddad B et al. 2014. Depth averaged models for fast landslide propagation: Mathematical, rheological and numerical aspects. Arch Comput Methods Eng 22(1): 67-104.

Pham BT, Tien Bui D, Prakash I, Dholakia M. 2017. Hybrid integration of multilayer perceptron neural networks and machine learning ensembles for landslide susceptibility assessment at Himalayan area (India) using GIS. Catena 149: 52-63.

Pilz M, Parolai S, Bindi D, Saponaro A, Abdybachaev U. 2013. Combining seismic noise techniques for landslide characterization. Pure Appl Geophys 171(8): 1729-1745.

Poiraud A. 2012. Les glissements de terrain dans le bassin du tertiaire volcanisé du Puy-en-Velay (Massif central, France). Caractérisation, facteurs de contrôle et cartographie de l'aléa. Thèse de doctorat de l'Université de Blaise Pascal de Clermont-Ferrand, Clermont-Ferrand.

Reichenbach P, Rossi M, Malamud BD, Mihir M, Guzzetti F. 2018. A review of statistically-based landslide susceptibility models. EarthSci Rev 180: 60-91.

Reninger PA. 2012. Méthodologie d'analyse de levés électromagnétiques aéroportés en domaine temporel pour la caractérisation géologique et hydrogéologique. Thèse de doctorat de l'Université d'Orléans, Orléans.

Reninger PA, Nachbaur A, Deparis J, Bernardie S. 2014. Exploitation de levés d'électromagnétisme héliporté dans une perspective de réévaluation des cartes d'aléa «mouvement de terrain » en milieu volcanique tropical - Application aux Antilles Phase 1. Disponible sur http://infoterre.brgm.fr/rapports/RP-64427-FR.pdf.

Saaty TL. 1977. A scaling method for priorities in hierarchical structures. J Math Psychol 15: 234-281.

Salciarini D, Godt JW, Savage WZ, Conversini P, Baum RL, Michael JA. 2006. Modeling regional initiation of rainfallinduced shallow landslides in the eastern Umbria Region of central Italy. Landslides 3: 181-194.

Samyn K, Bitri A, Courtois A. 2011. Tomographie 3D de glissement de terrain par traitement du bruit de fond sismique : application au glissement de terrain de la Valette. Rapport BRGM/RP-59845-FR, 65 p. Disponible sur http://infoterre.brgm.fr/rapports/RP-59845FR.pdf.

Santangelo M, Cardinali M, Rossi M, Mondini AC, Guzzetti F. 2010. Remote landslide mapping using a laser rangefinder binocular and GPS. Nat Hazard Earth Syst Sci 10: 2539-2546.

Sato HP, Yagi H, Moarai M, Iwahashi J, Sekiguchi T. Airborne lidar data measurement and landform classification mapping in Tomarino-tai landslide area, Shirakami Mountains, Japan. In: Sassa K, Fukuoka H, Wang F, Wang G, (eds). Progress in landslide science, Berlin: Springer, 2007, pp. 237-249.

Schlögel R, Doubre C, Malet JP, Masson F. 2015. Landslide deformation monitoring with ALOS/PALSAR imagery: A DInSAR geomorphological interpretation method. Geomorphology 231: 314-330, doi: 10.1016/j.geomorph.2014.11.031.

Sedan O. 2011. Logiciel ALICE V7. 0-Guide utilisateur. Rapport BRGM RP-60004-FR, 42 p. Disponible sur http://infoterre.brgm. fr/rapports/RP-60004-FR.pdf.

Soeters R, van Westen CJ. Slope instability, recognition, analysis, and zonation. In: Turner AK, Schuster RL, (eds). Landslides investigation and mitigation, transportation research board, Special 
Report 247. Washington: National Research Council, 1996, pp. 129-177.

Stumpf A, Kerle N. 2011. Combining Random Forests and object oriented analysis for landslide mapping from very high resolution imagery. Proced Environ Sci 3: 14-129.

Terrasol . 2018. TALREN-Manuel d'utilisation, 106 p. Disponible sur https://www.terrasol.fr/wp-content/uploads/2018/01/talren_v5_partie_c_-_fr.pdf.

Thiery Y. $\overline{2007}$. Susceptibilité du bassin de Barcelonnette (Alpes du Sud, France) aux mouvements de versant: cartographie morphodynamique, analyse spatiale et modélisation probabiliste. Thèse de doctorat de l'Université de Caen Basse-Normandie, Caen.

Thiery Y, Maquaire O, Fressard M. 2014. Application of expert rules in indirect approaches for landslide susceptibility assessment. Landslides 11: 411-424.

Thiery Y, Reninger PA, Nachbaur A. 2016. Exploitation de levés d'électromagnétisme héliporté dans une perspective de réévaluation des cartes d'aléa «mouvement de terrain» en milieu volcanique tropical. Rapport final. Disponible sur http://info terre.brgm.fr/rapports/RP-66605-FR.pdf.

Thiery Y, Lacquement F, Marçot N. 2019. Landslides triggered in weathered crystalline rocks of moderate latitudes: A case study in Mediterranean environment (The Maures Massif, France). Eng Geol 248: 164-184.

Thiery Y, Reninger PA, Nachbaur A, Vandromme R. 2015. Exploitation de levés d'électromagnétisme héliporté dans une perspective de réévaluation des cartes d'aléa «mouvement de terrain» en milieu volcanique tropical. Rapport intermédiaire. Disponible sur http://infoterre.brgm.fr/rapports/RP-65407-FR.pdf.

Thiery Y, Malet JP, Sterlacchini S, Puissant A, Maquaire O. 2005. Analyse spatiale de la susceptibilité des versants aux mouvements de terrain, comparaison de deux approches spatialisées par SIG. Rev Int Géomat/Eur J GIS Spat Anal 15: 227-245.

Thiery Y, Malet JP, Sterlacchini S, Puissant A, Maquaire O. 2007. Landslide susceptibility assessment by bivariate methods at large scales: Application to a complex mountainous environment. Geomorphology 9(1-2): 38-59.

Thiery Y, Vandromme R, Maquaire O, Bernardie S. Landslide susceptibility assessment by EPBM (Expert physically based model): Strategy of calibration in complex environment. In: Mikoš M, Tiwari B, Yin Y, Sassa K, (eds). Advances in landslide science,
Proceedings of the 4th World Landslide Forum, Ljubljana, Advancing Culture of Living with Landslides Volume 2, Slovenia, 29 May-2 June 2017, Berlin, Germany: Springer, 2017a, pp. 917 $-926$.

Thiery Y, Reninger PA, Lacquement F, Raingeard A, Lombard M, Nachbaur A. 2017b. Analysis of slope sensitivity to landslides by a transdisciplinary approach in the framework of future development: The case of La Trinité in Martinique (French West Indies). Geosciences 7(4): 135.

van den Eeckhaut M, Vanwalleghem T, Poesen J, Verstraeten G, Vandekerckhove L. 2006. Prediction landslide susceptibility using rare events logistic regression: A case-study in the Flemish Ardennes (Belgium). Geomorphology 76: 392-410.

van den Eeckhaut M, Poesen J, Verstraeten G et al. 2005. The use of LIDAR-derived images for mapping old landslides under forest. Earth Surf Process Landf 32: 754-769.

van Westen CJ, Rengers N, Soeters R. 2003. Use of geomorphological information in indirect landslide susceptibility assessment. Nat Hazard 30: 399-419.

van Westen CJ, Van Asch TWJ, Soeters R. 2006. Landslide hazard and risk zonation-Why is it still so difficult? Bull Eng Geol Environ 65: 167-184.

van Westen CJ, Castellanos E, Kuriakose SL. 2008. Spatial data for landslide susceptibility, hazard, and vulnerability assessment: An overview. Eng Geol 102(3): 112-131.

Varnes DJ. Slope movement types and processes. In: Schuster RL, Krizek RJ, (eds). Landslides: Analysis and control, Special Report 176. Washington, DC, USA: Transportation Research Board, National Research Council, 1978, pp. 11-33.

$\mathrm{Wu}$ TH, Tang WH, Einstein HH. Landslide hazard and risk assessment. In: Turner AK, Schuster RL, (eds). Landslides: Investigation and mitigation. Washington: National Academy Press, 1996, pp. 106-118.

Zêzere JL, Reis E, Garcia R et al. 2004. Integration of spatial and temporal data for the definition of different landslide hazard scenarios in the area north of Lisbon (Portugal). Nat Hazard Earth Syst Sci 4: 133-146.

Zizioli D, Meisina C, Valentino R, Montrasio L. 2013. Comparison between different approaches to modeling shallow landslide susceptibility: A case history in Oltrepo Pavese, Northern Italy. Nat Hazard Earth Syst Sci 13: 559-573.

Citation de l'article : Yannick Thiery, Monique Terrier. Évaluation de l'aléa glissements de terrain : état de l'art et perspectives pour la cartographie réglementaire en France. Rev. Fr. Geotech. 2018, 156, 3. 